\title{
Total Synthesis of Narbonolide and Biotransformation to
}

\section{Pikromycin}

Lakshmanan Venkatraman, ${ }^{\dagger}$ Christine E. Salomon, ${ }^{\ddagger}$ David H. Sherman, ${ }^{\S}$ and Robert A. Fecik ${ }^{* \dagger}$

${ }^{\dagger}$ Department of Medicinal Chemistry, 308 Harvard Street S.E., 8-101 WDH, University of Minnesota, Minneapolis, Minnesota 55455-0353, "Department of Microbiology, 420 Delaware Street S.E., University of Minnesota, Minneapolis, Minnesota 55455, and ${ }^{8}$ University of Michigan Life Sciences Institute, Departments of Medicinal Chemistry, Microbiology \& Immunology, and Chemistry, 210 Washtenaw Avenue, Ann Arbor, Michigan 48109-2216

fecik001@umn.edu

\section{Table of Contents}

General procedures S3

Experimental procedures and spectral data:

Aldol adduct 5 S3

Diol 6 S4

Ketal 7 S4

PMB ether 8 S5

Aldehyde 9 S5

Carboxylic acid $\mathbf{1 1}$ S6

Ester 13 S6

Alcohol 14 S7 
Aldehyde 15 S8

Allylic alcohol 16_ S8

Ketone 17 (from allylic alcohol 16)__ S9

Aldehyde 18_ S10

Ketone 17 (from diol 19)__ S10

${ }^{1} \mathrm{H}$ and ${ }^{13} \mathrm{C}$ NMR spectra:

Aldol adduct 5_ S12

Diol 6

Ketal 7__ S14

PMB ether 8

Aldehyde 9__ S16

Alcohol 10_ S17

Carboxylic acid $\mathbf{1 1}$ S18

Ester 13_ S19

Alcohol 14_ S20

Aldehyde 15__ S21

Allylic alcohol 16_ S22

Ketone 17_ S23

Narbonolide (1)__ S24

Aldehyde 18_ S25

Allylic alcohol 19_ S26

LC-MS and MS (ESI -) spectra:

Pikromycin (3)__ S27

References___ S28 
General Procedures. All commercial reagents were used as provided unless otherwise indicated. The Dess-Martin periodinane ${ }^{1}$ was synthesized according to the modified method of Ireland. $^{2} \mathrm{THF} \mathrm{Et}_{2} \mathrm{O}$, and $\mathrm{CH}_{2} \mathrm{Cl}_{2}$ were purified by passage through alumina columns. All reactions were performed under an inert atmosphere of dry $\mathrm{N}_{2}$ in oven-dried $\left(150^{\circ} \mathrm{C}\right)$ glassware. Optical rotations were determined on a polarimeter using the sodium D line $(\lambda=589 \mathrm{~nm})$ at the temperature indicated and are reported as follows: $[\alpha]_{\mathrm{D}}^{\text {temp }}$, concentration $(c=\mathrm{g} / 100 \mathrm{~mL})$, and solvent. Proton chemical shifts are reported in ppm from an internal standard of residual chloroform $(7.26 \mathrm{ppm})$, and carbon chemical shifts are reported using an internal standard of residual chloroform $(77.0 \mathrm{ppm})$. Proton chemical data are reported as follows: chemical shift, multiplicity (ovlp = overlapping, $\mathrm{s}=$ singlet, $\mathrm{d}=$ doublet, $\mathrm{t}=$ triplet, $\mathrm{q}=$ quartet, $\mathrm{p}=$ pentet, $\mathrm{m}=$ multiplet, $\mathrm{br}=$ broad $)$, coupling constant, and integration. High resolution mass spectra were obtained by ESI-TOF/MS using either PEG or PPG standards as high resolution calibrants. HPLC analyses were performed with a diode array and multiple wavelength detector. LC-MS data were obtained with a UV6000L diode array detector and an electrospray-ion trap mass spectrometer for ESI.

\section{(R)-3-((2S,3S,4S,6R)-3-Hydroxy-2,4,6-trimethyl-7-(triisopropylsilyloxy)heptanoyl)-4-}

benzyloxazolin-2-one (5). To a solution of $R$-4-benzyl-3-propionyl-2-oxazolidinone (2.33 g, $10 \mathrm{mmol}$, 1.0 equiv) in $\mathrm{CH}_{2} \mathrm{Cl}_{2}$ at $-78{ }^{\circ} \mathrm{C}$ was added freshly distilled $\mathrm{Bu}_{2} \mathrm{BOTf}(3.0 \mathrm{~mL}, 12 \mathrm{mmol}, 1.2$ equiv). After $15 \mathrm{~min}$, distilled $i$ - $\operatorname{Pr}_{2} \mathrm{NEt}(2.3 \mathrm{~mL}, 13.0 \mathrm{mmol}, 1.3$ equiv) was added, and the temperature was raised to $0{ }^{\circ} \mathrm{C}$ and stirred for $1 \mathrm{~h}$. The mixture was cooled back to $-78{ }^{\circ} \mathrm{C}$ and a solution of aldehyde $4^{3}$ (2.84 g, $10.0 \mathrm{mmol}, 1.0$ equiv) in $\mathrm{CH}_{2} \mathrm{Cl}_{2}(10 \mathrm{~mL})$ was added. The reaction mixture was stirred at $-78{ }^{\circ} \mathrm{C}$ for $30 \mathrm{~min}$, followed by stirring at $-10^{\circ} \mathrm{C}$ for $2 \mathrm{~h}$. The reaction was quenched by the addition of $\mathrm{pH} 7$ phosphate buffer $(1 \mathrm{M}, 10 \mathrm{~mL})$ and $\mathrm{MeOH}(25 \mathrm{~mL})$. The mixture was treated with a solution of $30 \%$ $\mathrm{H}_{2} \mathrm{O}_{2}(9 \mathrm{~mL})$ and $\mathrm{MeOH}(17 \mathrm{~mL})$ and extracted with $\mathrm{Et}_{2} \mathrm{O}(5 \times 15 \mathrm{~mL})$. Purification by flash chromatography (20\% EtOAc/hexanes) afforded the title compound (4.07 g, $78 \%$ yield) as a colorless oil. $R_{f}=0.46(20 \%$ EtOAc/hexanes $) ;[\alpha]_{\mathrm{D}}{ }^{23}=-40.56\left(c=0.53, \mathrm{CH}_{2} \mathrm{Cl}_{2}\right) ;{ }^{1} \mathrm{H} \mathrm{NMR}\left(\mathrm{CDCl}_{3}, 300 \mathrm{MHz}\right) \delta$ 7.24-7.37 (m, 3H), 7.19-7.22 (m, 2H), 4.64-4.72 (m, 1H), 4.15-4.24 (m, 2H), 3.96 (dq, J = 2.1, $6.9 \mathrm{~Hz}$, 
1H), 3.55-3.62 (m, 2H), $3.44(\mathrm{dd}, J=5.7,9.3 \mathrm{~Hz}, 1 \mathrm{H}), 3.27(\mathrm{dd}, J=3.6,13.5 \mathrm{~Hz}, 1 \mathrm{H}), 2.77(\mathrm{dd}, J=$ 9.3, 13.5 Hz, 2H), 1.64-1.84 (ovlp, 4H), 1.21 (d, $J=6.9 \mathrm{~Hz}, 3 \mathrm{H}), 1.03-1.16$ (ovlp, 21H), 0.95 (d, $J=$ $6.3 \mathrm{~Hz}, 3 \mathrm{H}), 0.90(\mathrm{~d}, J=6.6 \mathrm{~Hz}, 3 \mathrm{H}) ;{ }^{13} \mathrm{C} \mathrm{NMR}\left(\mathrm{CDCl}_{3}, 75 \mathrm{MHz}\right) \delta 177.7,153.1,135.3,129.6,129.2$, 127.6, 76.4, 68.5, 66.4, 55.6, 40.1, 38.2, 38.1, 34.4, 34.0, 19.4, 18.5, 16.6, 12.4, 9.9; HRMS calcd for $\left(\mathrm{C}_{29} \mathrm{H}_{49} \mathrm{NO}_{5} \mathrm{Si}+\mathrm{Na}^{+}\right): 542.3278$, found 542.3275.

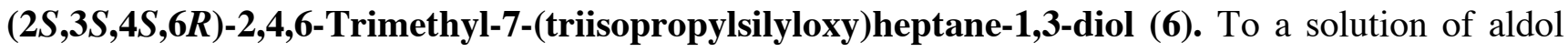
adduct $5(3.63 \mathrm{~g}, 7.0 \mathrm{mmol})$ in $\mathrm{THF}(35 \mathrm{~mL})$ at $0{ }^{\circ} \mathrm{C}$ was added $\mathrm{MeOH}(0.27 \mathrm{~mL})$ and $\mathrm{LiBH}_{4}(2 \mathrm{M}$ solution in THF, $3.5 \mathrm{~mL}, 7.0 \mathrm{mmol}, 1.0$ equiv), and the reaction was stirred for $45 \mathrm{~min}$. The reaction was quenched by the addition of saturated aqueous sodium potassium tartrate $(35 \mathrm{~mL})$ and stirred vigorously as it was warmed to room temperature. The reaction mixture was extracted with $\mathrm{CH}_{2} \mathrm{Cl}_{2}(3 \times$ $50 \mathrm{~mL})$. The combined organic extracts were washed with saturated aqueous $\mathrm{NaCl}(20 \mathrm{~mL})$, dried $\left(\mathrm{Na}_{2} \mathrm{SO}_{4}\right)$, filtered, and concentrated under reduced pressure. Purification by flash chromatography $(30 \%$ EtOAc/hexanes) afforded the title compound $\left(2.13 \mathrm{~g}, 88 \%\right.$ yield) as a colorless oil. $R_{f}=0.43(30 \%$ EtOAc/hexanes); $[\alpha]_{\mathrm{D}}{ }^{23}=+10.53\left(c=0.94, \mathrm{CH}_{2} \mathrm{Cl}_{2}\right) ;{ }^{1} \mathrm{H} \mathrm{NMR}\left(\mathrm{CDCl}_{3}, 300 \mathrm{MHz}\right) \delta 3.77(\mathrm{dd}, J=3.6$, $10.5 \mathrm{~Hz}, 1 \mathrm{H}), 3.68$ (dd, $J=5.4,10.5 \mathrm{~Hz}, 1 \mathrm{H}), 3.46-3.58$ (ovlp, 3H), 2.27 (br s, 2H), 1.58-1.88 (m, 4H), 1.03-1.14 (ovlp, 21H), 0.89-1.02 (ovlp, 7H), 0.84 (d, $J=7.2 \mathrm{~Hz}, 3 \mathrm{H}) ;{ }^{13} \mathrm{C} \mathrm{NMR}\left(\mathrm{CDCl}_{3}, 75 \mathrm{MHz}\right) \delta$ 80.3, 68.5, 68.4, 38.6, 36.3, 35.2, 34.3, 19.4, 18.4, 17.4, 12.4, 9.3; HRMS calcd for $\left(\mathrm{C}_{19} \mathrm{H}_{42} \mathrm{O}_{3} \mathrm{Si}+\mathrm{Na}^{+}\right)$: 369.2801, found 369.2804.

\section{$(2 R, 4 S)-4-\left(\left(2^{\prime} R, 4 ' S, 5 ' S\right)-2\right.$ '-(4-Methoxyphenyl)-5'-methyl-1',3'-dioxan-4-yl)-2-methyl-1-}

(triisopropylsilyloxy)pentane (7). To a solution of diol $6(2.13 \mathrm{~g}, 6.16 \mathrm{mmol})$ in $\mathrm{CH}_{2} \mathrm{Cl}_{2}(40 \mathrm{~mL})$ at room temperature was added $p$-anisaldehyde dimethylacetal (1.35 g, $7.4 \mathrm{mmol}, 1.2$ equiv) and CSA ( $0.07 \mathrm{~g}, 0.31 \mathrm{mmol}, 0.05$ equiv) and the reaction was stirred for $12 \mathrm{~h}$. The reaction was quenched by the addition of saturated aqueous $\mathrm{NaHCO}_{3}(15 \mathrm{~mL})$. The layers were separated and the aqueous layer was extracted with $\mathrm{CH}_{2} \mathrm{Cl}_{2}(3 \times 20 \mathrm{~mL})$. The combined organic layers were washed with saturated aqueous $\mathrm{NaCl}(20 \mathrm{~mL})$, dried $\left(\mathrm{MgSO}_{4}\right)$, filtered and concentrated under reduced pressure. Purification by flash 
chromatography (15\% EtOAc/hexanes) afforded the title compound (2.64 g, 92\% yield) as a colorless oil whose spectral data corresponded to those reported. ${ }^{4}$

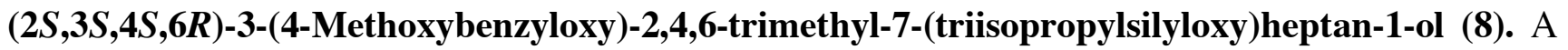
solution of cyclic acetal $7(1.86 \mathrm{~g}, 4.0 \mathrm{mmol})$ in toluene $(20 \mathrm{~mL})$ at $0{ }^{\circ} \mathrm{C}$ was treated dropwise with DIBAL-H (1.0 M solution in toluene, $12 \mathrm{~mL}, 12 \mathrm{mmol}, 3.0$ equiv) and stirred for $1 \mathrm{~h}$. The reaction was quenched by the addition of EtOAc $(12 \mathrm{~mL})$ and saturated aqueous sodium potassium tartrate $(12 \mathrm{~mL})$. The mixture was warmed to room temperature and stirred vigorously for 6 h. The mixture was extracted with $\mathrm{Et}_{2} \mathrm{O}(3 \times 30 \mathrm{~mL})$, and the combined organic layers were washed with saturated aqueous $\mathrm{NaCl}(10$ $\mathrm{mL})$, dried $\left(\mathrm{MgSO}_{4}\right)$, filtered, and concentrated under reduced pressure. Purification by flash chromatography (20\% EtOAc/hexanes) afforded the title compound (1.75 g, 94\% yield) as a colorless oil whose spectral data corresponded to those reported. ${ }^{4}$

(2S,3S,4S,6R)-3-(4-Methoxybenzyloxy)-2,4,6-trimethyl-7-(triisopropylsilyloxy)heptanal (9). To solution of $(\mathrm{COCl})_{2}(0.50 \mathrm{~mL}, 5.7 \mathrm{mmol}, 1.5$ equiv $)$ in $\mathrm{CH}_{2} \mathrm{Cl}_{2}(15 \mathrm{~mL})$ at $-78{ }^{\circ} \mathrm{C}$ was added dropwise a solution of DMSO (0.81 mL, $11.4 \mathrm{mmol}, 3.0$ equiv) in $\mathrm{CH}_{2} \mathrm{Cl}_{2}(5 \mathrm{~mL})$. After $15 \mathrm{~min}$ the cloudy solution was treated with a solution of alcohol $8(1.77 \mathrm{~g}, 3.8 \mathrm{mmol})$ in $\mathrm{CH}_{2} \mathrm{Cl}_{2}(25 \mathrm{~mL})$. The reaction was stirred for $15 \mathrm{~min}$ at $-78{ }^{\circ} \mathrm{C}$, then treated with $i-\operatorname{Pr}_{2} \mathrm{NEt}(3.3 \mathrm{~mL}, 19.0 \mathrm{mmol}, 5.0$ equiv). After stirring for 30 min at $-78{ }^{\circ} \mathrm{C}$, the reaction mixture was warmed to $0{ }^{\circ} \mathrm{C}$ and stirred for $1 \mathrm{~h}$. The reaction was quenched by the addition of saturated aqueous $\mathrm{NH}_{4} \mathrm{Cl}(20 \mathrm{~mL})$ and the mixture was slowly warmed to room temperature. The layers were separated and the aqueous layer was extracted with $\mathrm{CH}_{2} \mathrm{Cl}_{2}(3 \times 30 \mathrm{~mL})$. The combined organic layers were washed with saturated aqueous $\mathrm{NaCl}(15 \mathrm{~mL})$, dried $\left(\mathrm{Na}_{2} \mathrm{SO}_{4}\right)$, filtered, and concentrated under reduced pressure. Purification by flash chromatography (10\% EtOAc/hexanes) afforded the title compound $(1.52 \mathrm{~g}, 86 \%$ yield $)$ as a colorless oil. $R_{f}=0.53(10 \%$ EtOAc/hexanes); $[\alpha]_{\mathrm{D}}^{23}=-16.59\left(c=0.91, \mathrm{CH}_{2} \mathrm{Cl}_{2}\right) ;{ }^{1} \mathrm{H} \mathrm{NMR}\left(\mathrm{CDCl}_{3}, 300 \mathrm{MHz}\right) \delta 9.72(\mathrm{~s}, 1 \mathrm{H}), 7.20$ $(\mathrm{d}, J=8.7 \mathrm{~Hz}, 2 \mathrm{H}), 6.84(\mathrm{~d}, J=8.7 \mathrm{~Hz}, 2 \mathrm{H}), 4.38\left(\mathrm{AB}\right.$ system, $\left.J=10.8 \mathrm{~Hz}, \mathrm{v}_{\mathrm{AB}}=23.4,2 \mathrm{H}\right), 3.79(\mathrm{~s}$, 3H), $3.66(\mathrm{dd}, J=3.6,6.3 \mathrm{~Hz}, 1 \mathrm{H}), 3.53(\mathrm{dd}, J=5.1,6.3 \mathrm{~Hz}, 1 \mathrm{H}), 3.42(\mathrm{dd}, J=6.3,9.3 \mathrm{~Hz}, 1 \mathrm{H}), 2.53-$ 
2.62 (m, 1H), 1.95 (m, 1H), 1.52-1.75 (ovlp, 2H), 1.16 (d, J = 6.9 Hz, 3H), 1.02-1.09 (ovlp, 22H), 0.94

$(\mathrm{d}, J=6.6 \mathrm{~Hz}, 3 \mathrm{H}), 0.93(\mathrm{~d}, J=6.6 \mathrm{~Hz}, 3 \mathrm{H}) ;{ }^{13} \mathrm{C} \mathrm{NMR}\left(\mathrm{CDCl}_{3}, 75 \mathrm{MHz}\right) \delta 204.9,159.3,130.7,129.4$, $113.9,82.0,73.1,68.3,55.6,49.1,37.5,33.9,18.9,18.5,18.1,17.2,12.4,8.9$; HRMS calcd for $\left(\mathrm{C}_{27} \mathrm{H}_{48} \mathrm{O}_{4} \mathrm{Si}+\mathrm{Na}^{+}\right): 487.3220$, found 487.3218.

\section{(2R,3S,4S,5S,6S,8R)-3-Hydroxy-5-(4-methoxybenzyloxy)-2,4,6,8-tetramethyl-9-}

(triisopropylsilyloxy)nonanoic acid (11). To a solution of oxazolidinone $\mathbf{1 0}$ (1.05 g, $1.5 \mathrm{mmol})$ in THF $(16 \mathrm{~mL})$ and $\mathrm{H}_{2} \mathrm{O}(4 \mathrm{~mL})$ at $0{ }^{\circ} \mathrm{C}$ was added $30 \%$ aqueous $\mathrm{H}_{2} \mathrm{O}_{2}(1.7 \mathrm{~mL}, 15.0 \mathrm{mmol}, 10.0$ equiv) and aqueous $\mathrm{LiOH}(1 \mathrm{M}, 4.5 \mathrm{~mL}, 4.5 \mathrm{mmol}, 3.0$ equiv). After $1 \mathrm{~h}$ the solution was warmed to room temperature and stirred for an additional $6 \mathrm{~h}$. The solution was cooled to $0{ }^{\circ} \mathrm{C}$ and treated with aqueous $\mathrm{Na}_{2} \mathrm{SO}_{3}(1.5 \mathrm{M}, 10 \mathrm{~mL})$. After $10 \mathrm{~min}$, the mixture was diluted with EtOAc $(20 \mathrm{~mL})$ and acidified to $\mathrm{pH}$ 1 with $1 \mathrm{M} \mathrm{HCl}$. The layers were separated and the aqueous layer was extracted with EtOAc $(3 \times 20$ $\mathrm{mL})$. The combined organic layers were washed with saturated aqueous $\mathrm{NaCl}(10 \mathrm{~mL})$, dried $\left(\mathrm{Na}_{2} \mathrm{SO}_{4}\right)$, filtered, and concentrated under reduced pressure. Purification by flash chromatography (5\% $\left.\mathrm{MeOH} / \mathrm{CH}_{2} \mathrm{Cl}_{2}\right)$ afforded the title compound $(0.66 \mathrm{~g}, 82 \%$ yield $)$ as a colorless oil. $R_{f}=0.35(5 \%$ $\left.\mathrm{MeOH} / \mathrm{CH}_{2} \mathrm{Cl}_{2}\right) ;[\alpha]_{\mathrm{D}}{ }^{23}=+5.78\left(c=1.80, \mathrm{CH}_{2} \mathrm{Cl}_{2}\right) ;{ }^{1} \mathrm{H} \mathrm{NMR}\left(\mathrm{CDCl}_{3}, 300 \mathrm{MHz}\right) \delta 7.26(\mathrm{~d}, J=8.7 \mathrm{~Hz}$, 2H), $6.87(\mathrm{~d}, J=8.7 \mathrm{~Hz}, 2 \mathrm{H}), 4.52\left(\mathrm{AB}\right.$ system, $\left.J=10.7 \mathrm{~Hz}, v_{\mathrm{AB}} 75.3,2 \mathrm{H}\right), 3.93(\mathrm{dd}, J=2.4,8.4 \mathrm{~Hz}$, 1H), $3.80(\mathrm{~s}, 3 \mathrm{H}), 3.57(\mathrm{dd}, J=5.1,9.6 \mathrm{~Hz}, 1 \mathrm{H}), 3.47(\mathrm{dd}, J=5.7,9.6 \mathrm{~Hz}, 1 \mathrm{H}), 3.36(\mathrm{dd}, J=2.4,6.6$ Hz, 1H), 2.67 (pent, $J=7.2 \mathrm{~Hz}, 1 \mathrm{H}), 2.07-2.14(\mathrm{~m}, 1 \mathrm{H}), 1.87-1.94(\mathrm{~m}, 1 \mathrm{H}), 1.70-1.78(\mathrm{~m}, 1 \mathrm{H}), 1.58-$ $1.67(\mathrm{~m}, 1 \mathrm{H}), 1.25(\mathrm{~d}, J=6.9 \mathrm{~Hz}, 3 \mathrm{H}), 1.01-1.19(\mathrm{ovlp}, 23 \mathrm{H}), 0.97$ (d, $J=7.2 \mathrm{~Hz}, 3 \mathrm{H}), 0.96(\mathrm{~d}, J=6.3$ $\mathrm{Hz}, 3 \mathrm{H}), 0.95(\mathrm{~d}, J=6.6 \mathrm{~Hz}, 3 \mathrm{H}) ;{ }^{13} \mathrm{C} \mathrm{NMR}\left(\mathrm{CDCl}_{3}, 75 \mathrm{MHz}\right) \delta 180.5,159.4,130.4,129.6,114.1,87.9$, 76.7, 73.2, 68.3, 55.6, 43.3, 37.6, 37.0, 33.8, 33.3, 19.1, 18.5, 16.8, 13.8, 12.4, 8.1; HRMS calcd for $\left(\mathrm{C}_{30} \mathrm{H}_{54} \mathrm{O}_{6} \mathrm{Si}+\mathrm{Na}^{+}\right): 561.3587$, found 561.3593.

$(2 R, 3 S, 4 S, 5 S, 6 S, 8 R)-((3 R, 4 R, 5 E)-6-I o d o-4-m e t h y l h e x-5-e n-3-y l)$

3-hydroxy-5-(4methoxybenzyloxy)-2,4,6,8-tetramethyl-9-(triisopropylsilyloxy)nonanoate (13). To a solution of acid $11(0.81 \mathrm{~g}, 1.50 \mathrm{mmol})$ in THF $(15 \mathrm{~mL})$ at room temperature was added 2,4,6- 
trichlorobenzoylchloride $\left(0.25 \mathrm{~mL}, 1.65 \mathrm{mmol}, 1.1\right.$ equiv) and $\mathrm{Et}_{3} \mathrm{~N}(0.22 \mathrm{~mL}, 1.58 \mathrm{mmol}, 1.05$ equiv).

After stirring for $3 \mathrm{~h}$, the solids were filtered and washed with hexanes. The solvents were removed under reduced pressure and the residue was dissolved in benzene $(20 \mathrm{~mL})$. To this solution was added vinyliodide $12^{4}(0.43 \mathrm{~g}, 1.80 \mathrm{mmol}, 1.20$ equiv) in benzene (10 mL) and DMAP (0.25 g, $2.03 \mathrm{mmol}$, 1.35 equiv), and the reaction was stirred at room temperature for $24 \mathrm{~h}$. The reaction was diluted with $\mathrm{Et}_{2} \mathrm{O}(100 \mathrm{~mL})$, washed with saturated aqueous $\mathrm{NaHCO}_{3}(15 \mathrm{~mL})$ and saturated aqueous $\mathrm{NaCl}(10 \mathrm{~mL})$, dried $\left(\mathrm{MgSO}_{4}\right)$, filtered, and concentrated under reduced pressure. Purification by flash chromatography (20\% EtOAc/hexanes) afforded the title compound $(0.69 \mathrm{~g}, 61 \%$ yield $)$ as a colorless oil. $R_{f}=0.41(20 \%$ EtOAc/hexanes); $[\alpha]_{\mathrm{D}}^{23}=+28.80\left(c=1.00, \mathrm{CH}_{2} \mathrm{Cl}_{2}\right) ;{ }^{1} \mathrm{H} \mathrm{NMR}\left(\mathrm{CDCl}_{3}, 300 \mathrm{MHz}\right) \delta 7.25(\mathrm{~d}, J=8.4 \mathrm{~Hz}$, 2H), $6.87(\mathrm{~d}, J=8.7 \mathrm{~Hz}, 2 \mathrm{H}), 6,43(\mathrm{dd}, J=7.8,14.7 \mathrm{~Hz}, 1 \mathrm{H}), 6.08(\mathrm{dd}, J=0.9,14.7 \mathrm{~Hz}, 1 \mathrm{H}), 4.69-4.76$ $(\mathrm{m}, 1 \mathrm{H}), 4.52\left(\mathrm{AB}\right.$ system, $\left.J=10.5 \mathrm{~Hz}, v_{\mathrm{AB}} 66.3,2 \mathrm{H}\right), 3.88(\mathrm{dd}, J=2.4,8.7 \mathrm{~Hz}, 1 \mathrm{H}), 3.79(\mathrm{~s}, 3 \mathrm{H}), 3.58$ $(\mathrm{dd}, J=4.8,9.6 \mathrm{~Hz}, 1 \mathrm{H}), 3.45(\mathrm{dd}, J=6.3,9.6 \mathrm{~Hz}, 1 \mathrm{H}), 3.36(\mathrm{dd}, J=2.4,6.9 \mathrm{~Hz}, 1 \mathrm{H}), 2.59-2.69(\mathrm{~m}$, 1H), 2.42-2.48 (m, 1H), 2.03-2.10 (m, 1H), 1.44-1.82 (ovlp, 6H), 1.27 (d, J = 6.9 Hz, 3H), 1.03-1.11 (ovlp, 22H), 0.96-1.01 (ovlp, 6H), 0.95 (d, $J=6.6 \mathrm{~Hz}, 3 \mathrm{H}), 0.92$ (d, $J=7.2 \mathrm{~Hz}, 3 \mathrm{H}), 0.86(\mathrm{t}, J=7.5 \mathrm{~Hz}$, $3 \mathrm{H}) ;{ }^{13} \mathrm{C} \mathrm{NMR}\left(\mathrm{CDCl}_{3}, 75 \mathrm{MHz}\right) \delta 175.1,159.3,147.4,130.4,129.4,114.1,88.4,77.2,77.1,76.4,72.9$, 68.1, 55.6, 44.3, 43.9, 37.7, 37.2, 33.7, 33.3, 24.6, 19.2, 18.5, 16.5, 15.2, 14.8, 12.4, 10.3, 7.9; HRMS calcd for $\left(\mathrm{C}_{37} \mathrm{H}_{65} \mathrm{IO}_{6} \mathrm{Si}+\mathrm{Na}^{+}\right)$: 783.3493, found 783.3513.

\section{$(2 R, 3 S, 4 S, 5 S, 6 S, 8 R)-((3 R, 4 R, 5 E)-6-I o d o-4-m e t h y l h e x-5-e n-3-y l)$}

3,9-dihydroxy-5-(4-

methoxybenzyloxy)-2,4,6,8-tetramethylnonanoate (14). To a solution of silyl ether 13 (0.27 g, 0.355 mmol) in pyridine $(1.00 \mathrm{~mL})$ and THF $(15 \mathrm{~mL})$ was added $\mathrm{HF} \bullet$ pyridine $(70 \% \mathrm{HF}$ in pyridine, $2.00 \mathrm{~mL})$. The reaction was stirred for $48 \mathrm{~h}$ at room temperature, diluted with $\mathrm{Et}_{2} \mathrm{O}(50 \mathrm{~mL})$, washed with $\mathrm{H}_{2} \mathrm{O}(2 \times$ $5 \mathrm{~mL})$, saturated aqueous $\mathrm{NaCl}(5 \mathrm{~mL})$, dried $\left(\mathrm{MgSO}_{4}\right)$, filtered, and concentrated under reduced pressure. Purification by flash chromatography (40\% EtOAc/hexanes) afforded the title compound (0.17 g, 79\% yield) as a colorless oil. $R_{f}=0.51(40 \%$ EtOAc/hexanes $) ;[\alpha]_{\mathrm{D}}{ }^{23}=+40.63\left(c=0.48, \mathrm{CH}_{2} \mathrm{Cl}_{2}\right) ;{ }^{1} \mathrm{H}$ $\operatorname{NMR}\left(\mathrm{CDCl}_{3}, 300 \mathrm{MHz}\right) \delta 7.24(\mathrm{~d}, J=8.4 \mathrm{~Hz}, 2 \mathrm{H}), 6.84(\mathrm{~d}, J=8.4 \mathrm{~Hz}, 2 \mathrm{H}), 6.47(\mathrm{dd}, J=8.1,14.4 \mathrm{~Hz}$, 
1H), $6.06(\mathrm{~d}, J=14.4 \mathrm{~Hz}, 1 \mathrm{H}), 4.73-4.79(\mathrm{~m}, 1 \mathrm{H}), 4.55(\mathrm{~s}, 2 \mathrm{H}), 4.07$ (dd, $J=2.7,9.3 \mathrm{~Hz}, 1 \mathrm{H}), 3.77$ (s, 3H), 3.49 (dd, $J=4.5,10.5 \mathrm{~Hz}, 1 \mathrm{H}), 3.31-3.40$ (ovlp, 2H), 2.57-2.65 (m, 1H), 2.46 (sext, $J=6.6 \mathrm{~Hz}$, $1 \mathrm{H}), 1.41-1.91(\mathrm{~m}, 8 \mathrm{H}), 1.12(\mathrm{~d}, J=7.2 \mathrm{~Hz}, 3 \mathrm{H}), 1.03(\mathrm{~d}, J=6.6 \mathrm{~Hz}, 3 \mathrm{H}), 0.99(\mathrm{~d}, J=6.9 \mathrm{~Hz}, 3 \mathrm{H})$, 0.97-0.99 (m, 1H), $0.94(\mathrm{~d}, J=6.6 \mathrm{~Hz}, 3 \mathrm{H}), 0.83-0.84(\mathrm{ovlp}, 6 \mathrm{H}) ;{ }^{13} \mathrm{C} \mathrm{NMR}\left(\mathrm{CDCl}_{3}, 75 \mathrm{MHz}\right) \delta 175.3$, $159.4,147.7,130.2,129.7,114.1,89.7,77.4,76.2,74.9,74.6,67.7,55.6,44.3,42.6,38.4,35.9,34.5$, 33.7, 24.5, 18.8, 15.5, 15.4, 10.3, 9.1; HRMS calcd for $\left(\mathrm{C}_{28} \mathrm{H}_{45} \mathrm{IO}_{6}+\mathrm{Na}^{+}\right)$: 627.2159, found 627.2183.

$(2 R / S, 3 S, 4 S, 5 S, 6 S, 8 R)-((3 R, 4 R, E)-6-$ Iodo-4-methylhex-5-en-3-yl)

3-hydroxy-5-(4methoxybenzyloxy)-2,4,6,8-tetramethyl-9-oxononanoate (15). To a solution of alcohol 14 (0.17 g, $0.282 \mathrm{mmol})$ in $\mathrm{CH}_{2} \mathrm{Cl}_{2}(30 \mathrm{~mL})$ at room temperature was added the Dess-Martin periodinane (0.48 $\mathrm{g}$, $1.126 \mathrm{mmol}, 4.0$ equiv) and pyridine $(1.25 \mathrm{~mL})$. The reaction was stirred for $12 \mathrm{~h}$ and diluted with EtOAc $(100 \mathrm{~mL})$. The mixture was washed with saturated aqueous $\mathrm{NaHCO}_{3}(20 \mathrm{~mL}), 1 \mathrm{M}$ aqueous $\mathrm{Na}_{2} \mathrm{SO}_{3}(20 \mathrm{~mL})$, and saturated aqueous $\mathrm{NaCl}(20 \mathrm{~mL})$, dried $\left(\mathrm{Na}_{2} \mathrm{SO}_{4}\right)$, filtered, and concentrated under reduced pressure. Purification by flash chromatography (15\% EtOAc/hexanes) afforded the title compound $(0.131 \mathrm{~g}, 77 \%$ yield $)$ as a colorless oil. $R_{f}=0.51(15 \%$ EtOAc/hexanes $) ;{ }^{1} \mathrm{H} \mathrm{NMR}\left(\mathrm{CDCl}_{3}\right.$, $300 \mathrm{MHz}) \delta 9.56(\mathrm{~s}, 1 \mathrm{H}), 7.14(\mathrm{~d}, J=8.4 \mathrm{~Hz}, 2 \mathrm{H}), 6.82(\mathrm{~d}, J=8.7 \mathrm{~Hz}, 2 \mathrm{H}), 6.39(\mathrm{dd}, J=8.4,14.7 \mathrm{~Hz}$, $1 \mathrm{H}), 6.07(\mathrm{~d}, J=14.1 \mathrm{~Hz}, 1 \mathrm{H}), 4.72-4.78(\mathrm{~m}, 1 \mathrm{H}), 4.32\left(\mathrm{AB}\right.$ system, $\left.J=10.8 \mathrm{~Hz}, v_{\mathrm{AB}} 49.2,2 \mathrm{H}\right), 3.78(\mathrm{~s}$, $3 \mathrm{H}), 3.66-3.71(\mathrm{~m}, 1 \mathrm{H}), 3.52(\mathrm{dd}, J=1.5,9.6 \mathrm{~Hz}, 1 \mathrm{H}), 3.04-3.15(\mathrm{~m}, 1 \mathrm{H}), 2.42-2.51(\mathrm{~m}, 2 \mathrm{H}), 1.72-$ $1.88(\mathrm{~m}, 2 \mathrm{H}), 1.29-1.63$ (ovlp, 3H), $1.20(\mathrm{~d}, J=6.9 \mathrm{~Hz}, 3 \mathrm{H}), 1.12(\mathrm{~d}, J=7.2 \mathrm{~Hz}, 3 \mathrm{H}), 1.05(\mathrm{~d}, J=6.6$ $\mathrm{Hz}, 3 \mathrm{H}), 1.04(\mathrm{~d}, J=6.6 \mathrm{~Hz}, 3 \mathrm{H}), 0.98(\mathrm{~d}, J=6.9 \mathrm{~Hz}, 3 \mathrm{H}), 0.85(\mathrm{t}, J=7.2 \mathrm{~Hz}, 3 \mathrm{H}) ;{ }^{13} \mathrm{C} \mathrm{NMR}\left(\mathrm{CDCl}_{3}\right.$, $75 \mathrm{MHz}) \delta 209.3,204.9,170.6,159.3,147.1,130.7,129.4,113.9,87.1,78.4,76.7,74.9,55.6,54.7$, 48.4, 44.4, 44.2, 44.0, 33.1, 32.1, 24.4, 18.1, 15.5, 15.2, 14.7, 12.8, 10.3; HRMS calcd for $\left(\mathrm{C}_{28} \mathrm{H}_{41} \mathrm{IO}_{6}+\right.$ $\mathrm{Na}^{+}$): 623.1846, found 623.1829.

(2R/S,9R/S)-9-Dihydro-5-(4-methoxybenzyloxy)narbonolide (16). To a solution of aldehyde 15 (130 mg, $0.2167 \mathrm{mmol})$ in DMSO $(87 \mathrm{~mL})$ at room temperature was added $\mathrm{CrCl}_{2}(266 \mathrm{mg}, 2.1670$ mmol, 10 equiv) and $\mathrm{NiCl}_{2}(2.7 \mathrm{mg}, 0.0217 \mathrm{mmol}, 0.1$ equiv). The reaction was stirred for $12 \mathrm{~h}$, then 
quenched by the addition of $\mathrm{H}_{2} \mathrm{O}(40 \mathrm{~mL})$. The mixture was diluted with EtOAc $(500 \mathrm{~mL})$ and the layers were separated. The organic layer was washed with $\mathrm{H}_{2} \mathrm{O}(3 \times 50 \mathrm{~mL})$ and saturated aqueous $\mathrm{NaCl}(20$ $\mathrm{mL})$, dried $\left(\mathrm{Na}_{2} \mathrm{SO}_{4}\right)$, filtered, and concentrated under reduced pressure. Purification by flash chromatography (30\% EtOAc/hexanes) afforded the title compound (91.0 $\mathrm{mg}, 89 \%$ yield) as a moist solid. $R_{f}=0.38\left(30 \%\right.$ EtOAc/hexanes); ${ }^{1} \mathrm{H}$ NMR $\left(\mathrm{CDCl}_{3}, 300 \mathrm{MHz}\right) \delta 7.11(\mathrm{~d}, J=8.1 \mathrm{~Hz}, 2 \mathrm{H}), 6.83(\mathrm{~d}$, $J=8.4 \mathrm{~Hz}, 2 \mathrm{H}), 5.62-5.79(\mathrm{~m}, 2 \mathrm{H}), 4.94-4.99(\mathrm{~m}, 1 \mathrm{H}), 4.27$ (AB system, $\left.J=10.5 \mathrm{~Hz}, v_{\mathrm{AB}} 42.3,2 \mathrm{H}\right)$, 4.04-4.06 (m, 1H), $3.78(\mathrm{~s}, 3 \mathrm{H}), 3.51(\mathrm{q}, J=6.6 \mathrm{~Hz}, 1 \mathrm{H}), 3.31-3.34(\mathrm{~m}, 1 \mathrm{H}), 2.82-2.92(\mathrm{~m}, 1 \mathrm{H}), 2.55$ (br s, 1H), 1.47-1.81 (ovlp, 7H), $1.19(\mathrm{~d}, J=6.9 \mathrm{~Hz}, 3 \mathrm{H}), 1.11(\mathrm{~d}, J=6.9 \mathrm{~Hz}, 3 \mathrm{H}), 1.09(\mathrm{~d}, J=7.2 \mathrm{~Hz}$ $3 \mathrm{H}), 1.07(\mathrm{~d}, J=6.9 \mathrm{~Hz}, 3 \mathrm{H}), 1.01(\mathrm{~d}, J=6.6 \mathrm{~Hz}, 3 \mathrm{H}), 0.89(\mathrm{t}, J=7.2 \mathrm{~Hz}, 3 \mathrm{H}) ;{ }^{13} \mathrm{C} \mathrm{NMR}\left(\mathrm{CDCl}_{3}, 75\right.$ MHz) $\delta 208.9,208.7,170.0,169.9,159.2,135.3,133.2,133.1,131.1,131.0,130.7,129.5,113.9,80.6$, $80.5,79.5,79.1,78.5,75.3,73.1,72.5,55.6,54.1,52.1,51.4,47.7,46.8,40.1,39.2,36.8,36.6,34.8$ $33.8,31.9,29.6,22.3,21.2,17.5,17.2,17.1,16.9,16.7,15.6,14.9,14.4,14.3,11.4,11.2 ;$ HRMS calcd for $\left(\mathrm{C}_{28} \mathrm{H}_{42} \mathrm{O}_{6}+\mathrm{Na}^{+}\right): 497.2879$, found 497.2853.

5-(4-Methoxybenzyloxy)narbonolide (17). To a solution of allylic alcohol 16 (28 mg, $0.059 \mathrm{mmol})$ in $\mathrm{CH}_{2} \mathrm{Cl}_{2}(5 \mathrm{~mL})$ at room temperature was added the Dess-Martin periodinane $(75 \mathrm{mg}, 0.177 \mathrm{mmol}, 4.0$ equiv) and pyridine $(0.15 \mathrm{~mL})$. The reaction was stirred for $18 \mathrm{~h}$ and diluted with EtOAc $(40 \mathrm{~mL})$. The mixture was washed with saturated aqueous $\mathrm{NaHCO}_{3}(5 \mathrm{~mL}), 1 \mathrm{M}$ aqueous $\mathrm{Na}_{2} \mathrm{SO}_{3}(5 \mathrm{~mL})$, and saturated aqueous $\mathrm{NaCl}(5 \mathrm{~mL})$, dried $\left(\mathrm{Na}_{2} \mathrm{SO}_{4}\right)$, filtered, and concentrated under reduced pressure. Purification by flash chromatography (20\% EtOAc/hexanes) afforded the title compound (24.7 $\mathrm{mg}, 88 \%$ yield $)$ as a moist solid. $R_{f}=0.56(20 \%$ EtOAc/hexanes $) ;[\alpha]_{\mathrm{D}}{ }^{23}=+86.44\left(c=0.59, \mathrm{CH}_{2} \mathrm{Cl}_{2}\right) ;{ }^{1} \mathrm{H} \mathrm{NMR}$ $\left(\mathrm{CDCl}_{3}, 300 \mathrm{MHz}\right) \delta 7.27(\mathrm{~d}, J=8.4 \mathrm{~Hz}, 2 \mathrm{H}), 6.87(\mathrm{~d}, J=8.1 \mathrm{~Hz}, 2 \mathrm{H}), 6.74(\mathrm{dd}, J=6.0,15.6 \mathrm{~Hz}, 1 \mathrm{H})$, $6.13(\mathrm{~d}, J=15.9 \mathrm{~Hz}, 1 \mathrm{H}), 4.91-4.94(\mathrm{~m}, 1 \mathrm{H}), 4.48(\mathrm{~s}, 2 \mathrm{H}), 3.89$ (d, $J=6.3 \mathrm{~Hz}, 1 \mathrm{H}), 3.79-3.82$ (ovlp, 4H), 2.84 (pent, $J=7.2 \mathrm{~Hz}, 1 \mathrm{H}), 2.58-2.69$ (m, 2H), 1.48-1.66 (ovlp, 4H), 1.35 (d, $J=7.2 \mathrm{~Hz}, 3 \mathrm{H})$, $1.28(\mathrm{~d}, J=7.2 \mathrm{~Hz}, 3 \mathrm{H}), 1.12-1.24(\mathrm{~m}, 1 \mathrm{H}), 1.10(\mathrm{~d}, J=7.5 \mathrm{~Hz}, 3 \mathrm{H}), 1.07(\mathrm{~d}, J=6.9,3 \mathrm{H}), 1.01(\mathrm{~d}, J=$ $6.6 \mathrm{~Hz}, 3 \mathrm{H}), 0.90(\mathrm{t}, J=7.2 \mathrm{~Hz}, 3 \mathrm{H}) ;{ }^{13} \mathrm{C} \mathrm{NMR}\left(\mathrm{CDCl}_{3}, 75 \mathrm{MHz}\right) \delta 208.2,203.2,169.6,159.3,147.8$, 
$130.9,129.5,127.1,113.9,79.8,78.7,74.0,55.6,51.0,49.6,43.3,38.8,36.2,23.6,18.2,16.4,14.9$,

14.7, 12.5, 10.9; HRMS calcd for $\left(\mathrm{C}_{28} \mathrm{H}_{40} \mathrm{O}_{6}+\mathrm{Na}^{+}\right)$: 495.2723, found 495.2727.

$(2 R, 3 S, 4 S, 5 S, 6 S, 8 R)-((3 R, 4 R, E)-6-I o d o-4-m e t h y l h e x-5-e n-3-y l)$

3-hydroxy-5-(4-

methoxybenzyloxy)-2,4,6,8-tetramethyl-9-oxononanoate (18). To a solution of diol 14 (30.2 mg, 0.05

mmol) in $\mathrm{CH}_{2} \mathrm{Cl}_{2}(5 \mathrm{~mL})$ was added saturated aqueous $\mathrm{NaHCO}_{3}(2 \mathrm{~mL})$ and a catalytic amount of $\mathrm{KBr}$.

The mixture was cooled to $0{ }^{\circ} \mathrm{C}$, a catalytic amount of TEMPO was added, and was stirred vigorously

and treated with aqueous $\mathrm{NaOCl}(0.25 \mathrm{M}, 0.22 \mathrm{~mL}, 0.055 \mathrm{mmol}, 1.1$ equiv) and stirred for $20 \mathrm{~min}$. The reaction was quenched by the addition of saturated aqueous $\mathrm{Na}_{2} \mathrm{SO}_{3}(1 \mathrm{~mL})$ and saturated aqueous $\mathrm{NaHCO}_{3}(10 \mathrm{~mL})$. The mixture was extracted with EtOAc $(3 \times 10 \mathrm{~mL})$ and the combined organic layers were dried $\left(\mathrm{MgSO}_{4}\right)$, filtered, and concentrated under reduced pressure. Purification by flash chromatography (30\% EtOAc/hexanes) afforded the title compound (28 $\mathrm{mg}, 93 \%$ yield) as a colorless oil. $R_{f}=0.61(30 \%$ EtOAc/hexanes $) ;[\alpha]_{\mathrm{D}}{ }^{23}=+40.42\left(c=0.48, \mathrm{CH}_{2} \mathrm{Cl}_{2}\right) ;{ }^{1} \mathrm{H} \mathrm{NMR}\left(\mathrm{CDCl}_{3}, 300 \mathrm{MHz}\right) \delta$ $9.54(\mathrm{~s}, 1 \mathrm{H}), 7.26(\mathrm{~d}, J=8.4 \mathrm{~Hz}, 2 \mathrm{H}), 6.87(\mathrm{~d}, J=8.4 \mathrm{~Hz}, 2 \mathrm{H}), 6.43(\mathrm{dd}, J=4.8,14.4 \mathrm{~Hz}, 1 \mathrm{H}), 6.09(\mathrm{~d}$, $J=14.4 \mathrm{~Hz}, 1 \mathrm{H}), 4.70-4.76(\mathrm{~m}, 1 \mathrm{H}), 4.53\left(\mathrm{AB}\right.$ system, $\left.J=10.5 \mathrm{~Hz}, v_{\mathrm{AB}} 30.3,2 \mathrm{H}\right) 3.86-3.89(\mathrm{~m}, 1 \mathrm{H})$, $3.80(\mathrm{~s}, 3 \mathrm{H}), 3.36(\mathrm{dd}, J=3.0,6.0 \mathrm{~Hz}, 1 \mathrm{H}), 3.03(\mathrm{~s}, 1 \mathrm{H}), 2.66$ (pent, $J=6.9 \mathrm{~Hz}, 1 \mathrm{H}), 2.39-2.52(\mathrm{~m}, 2 \mathrm{H})$, 1.75-2.07 (m, 3H), 1.42-1.66 (ovlp, 2H), 1.27 (d, $J=7.2 \mathrm{~Hz}, 3 \mathrm{H}), 1.15-1.22(\mathrm{~m}, 1 \mathrm{H}), 1.11(\mathrm{~d}, J=6.9$ $\mathrm{Hz}, 3 \mathrm{H}), 0.99(\mathrm{~d}, J=6.9 \mathrm{~Hz}, 3 \mathrm{H}), 0.98(\mathrm{~d}, J=7.2 \mathrm{~Hz}, 3 \mathrm{H}), 0.94(\mathrm{~d}, J=6.9 \mathrm{~Hz}, 3 \mathrm{H}), 0.86(\mathrm{t}, J=7.2 \mathrm{~Hz}$, $3 \mathrm{H}) ;{ }^{13} \mathrm{C} \mathrm{NMR}\left(\mathrm{CDCl}_{3}, 75 \mathrm{MHz}\right) \delta$ 205.2, 175.2, 159.4, 147.4, 130.2, 129.5, 114.2, 87.6, 77.3, 76.5, $76.4,73.6,55.6,44.4,44.1,37.6,34.5,33.5,24.6,16.7,15.3,15.2,14.8,10.3,8.1$; HRMS calcd for $\left(\mathrm{C}_{28} \mathrm{H}_{43} \mathrm{IO}_{6}+\mathrm{Na}^{+}\right): 625.2002$, found 625.2010.

5-(4-Methoxybenzyloxy)narbonolide (17). To a solution of diol 19 (20.0 $\mathrm{mg}, 0.042 \mathrm{mmol})$ in $\mathrm{CH}_{2} \mathrm{Cl}_{2}(8 \mathrm{~mL})$ at room temperature was added the Dess-Martin periodinane $(71.2 \mathrm{mg}, 0.168 \mathrm{mmol}, 4.0$ equiv). The reaction was stirred for $4 \mathrm{~h}$ and diluted with EtOAc $(40 \mathrm{~mL})$. The mixture was washed with saturated aqueous $\mathrm{NaHCO}_{3}(5 \mathrm{~mL}), 1 \mathrm{M} \mathrm{Na}_{2} \mathrm{SO}_{3}(5 \mathrm{~mL})$, and saturated aqueous $\mathrm{NaCl}(5 \mathrm{~mL})$, dried $\left(\mathrm{Na}_{2} \mathrm{SO}_{4}\right)$, filtered, and concentrated under reduced pressure. Purification by flash chromatography (20\% 
EtOAc/hexanes) afforded the title compound (17.6 mg, $88 \%$ yield) as a moist solid whose spectral data corresponded to those reported above. 


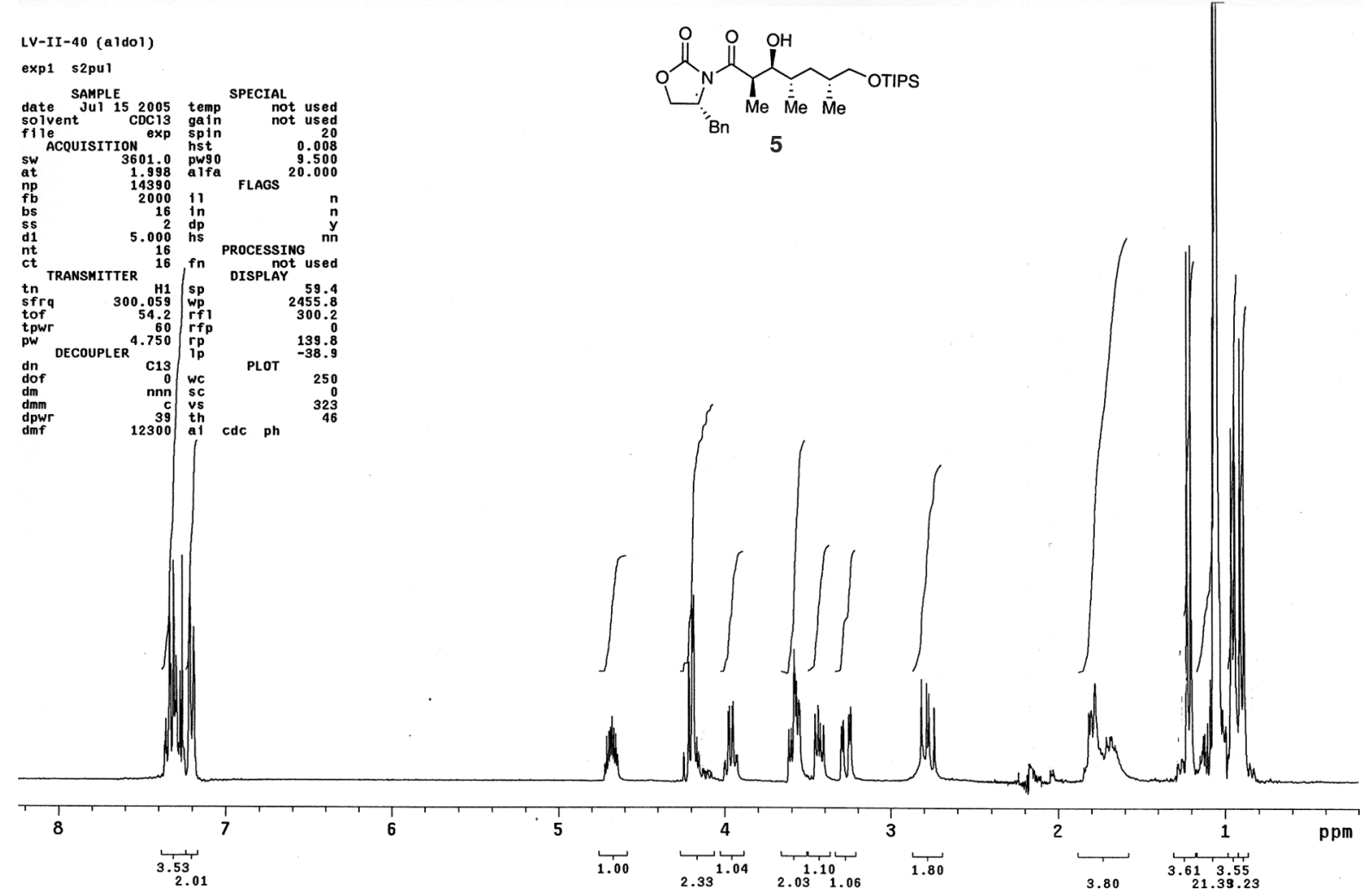

LV-II-40 (a)dol)

Archlve directory: /data/venkat/unmrsys/data
Sample directory: II-40_15Ju12005

File: CARBON

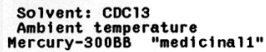

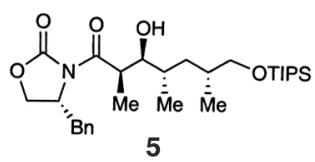

Re lax. delay $1.000 \mathrm{sec}$

cca time 1.815 se

BOSERVE C13 75.4495244 HHZ
DECOUPLE H1, 300.0590418 HHE

WALTZ-16 modulated
DATA PROCESSING

Line broadening $1.0 \mathrm{~Hz}$

Total time $48 \mathrm{~min}, 47 \mathrm{sec}$

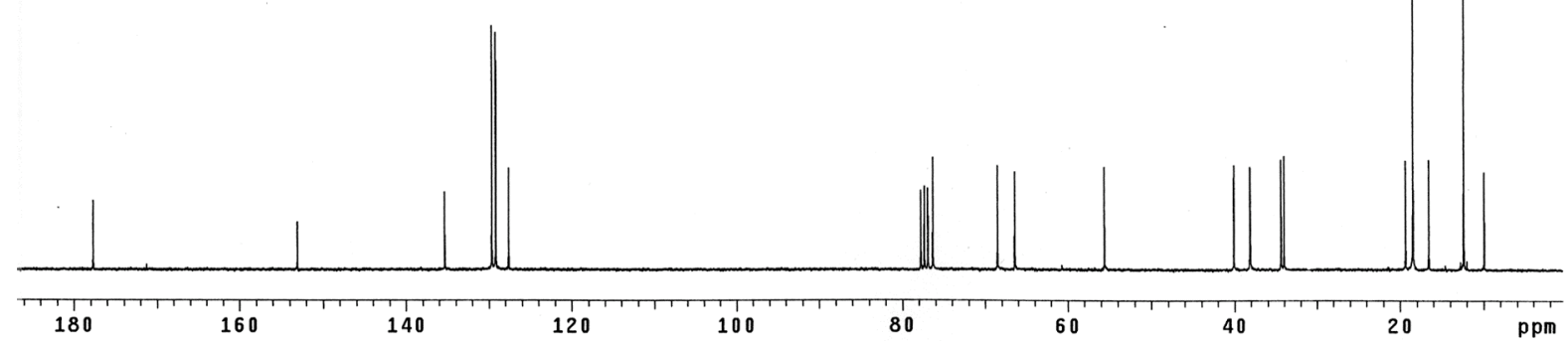



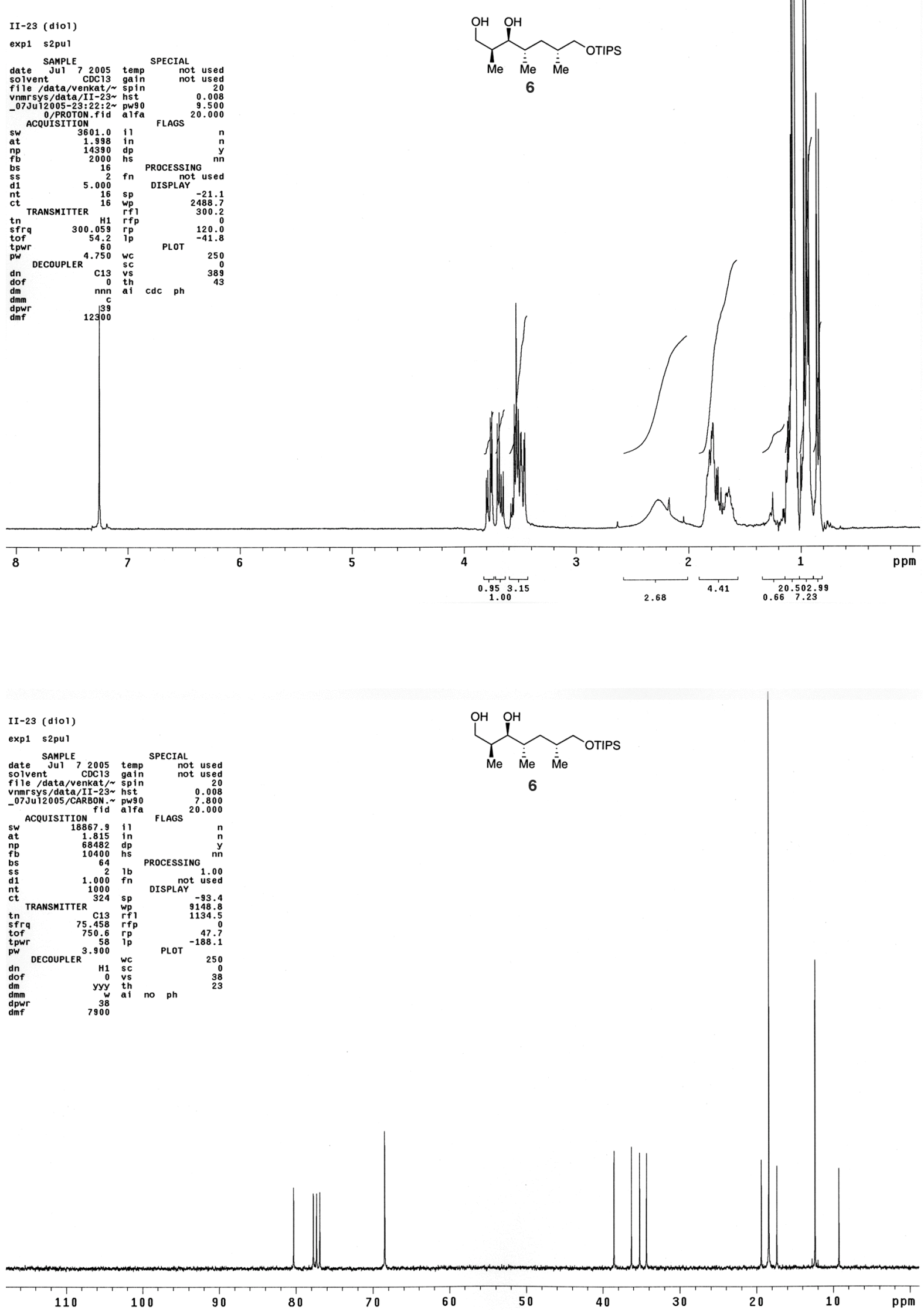

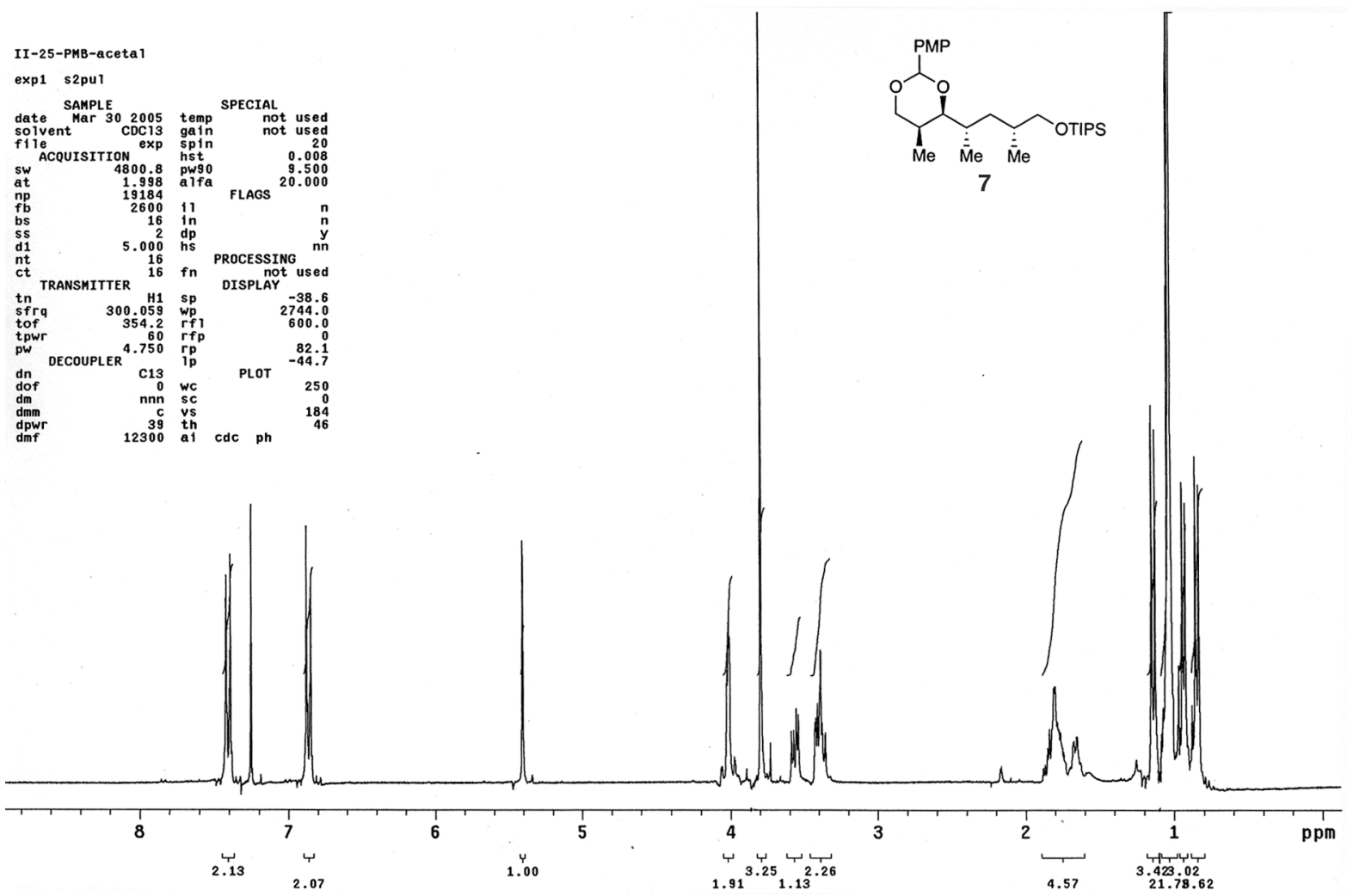

LV-II-42

Archlve directory: /data/venkat/unmrsys/data
Sample directory: LV-II-42-06Jun2005-15:16:49
File: CARBON

Pulse Sequence: s2pul

Solvent: $\mathrm{CDC}_{13}$

Solvent: CDCl3
Ambient temperature
Mercury-300BB "medicinal1"

Re lax. de lay $1.000 \mathrm{sec}$

Acq. $t$ ime 1.815 sec

Width 18867.99

OBSERVE C13, 75.4495244 MHZ

Power $38 \mathrm{~dB}$
cont inuous ly on

WALTZ-16 modula

Line broadening $1.0 \mathrm{~Hz}$
FT size $131072 \mathrm{~min}, 5 \mathrm{sec}$

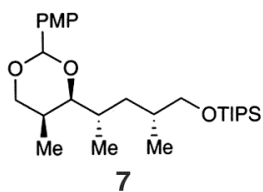

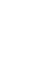

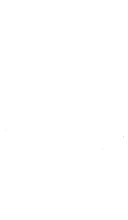



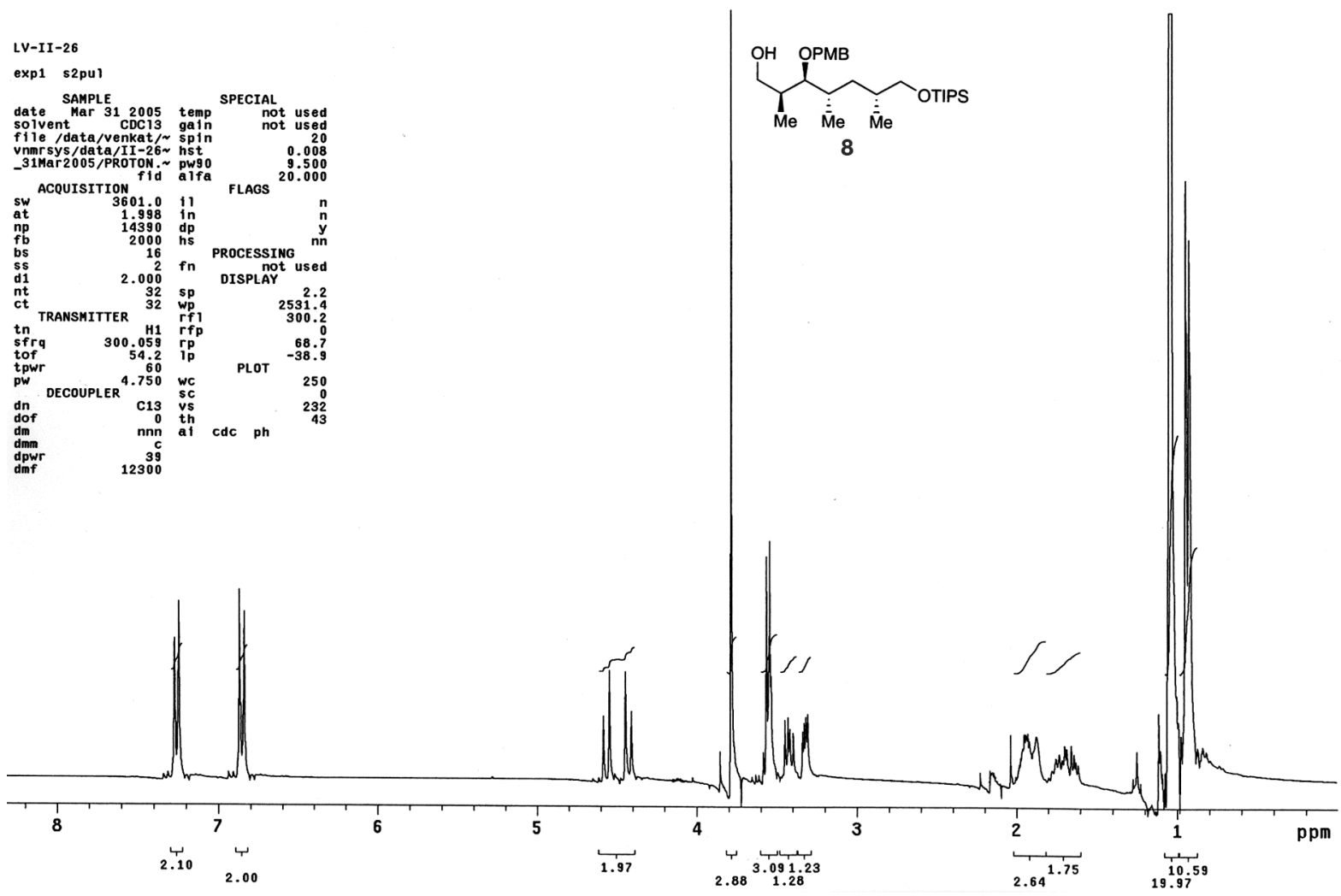

LV-II-26

Archive directory: /data/venkat/vnmrsys/data
Sample directory: II-26_31Mar2005

Pulse Sequence: s2pur

Solvent: CDC13

Ambient temperature
Mercury-300BB "medicinal1"

Re lax. delay $1.000 \mathrm{sec}$
Pulse 45.0 degrees

width $18867.9 \mathrm{~Hz}$

512 repetitions

DECOUPLE H1, 300.0590418 MH

Power 38 dB

WALTZ-16 modu lated

Line broadening $1.0 \mathrm{~Hz}$

Total time $25 \mathrm{~min}, 5 \mathrm{sec}$

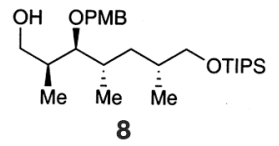

.

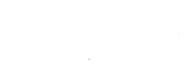




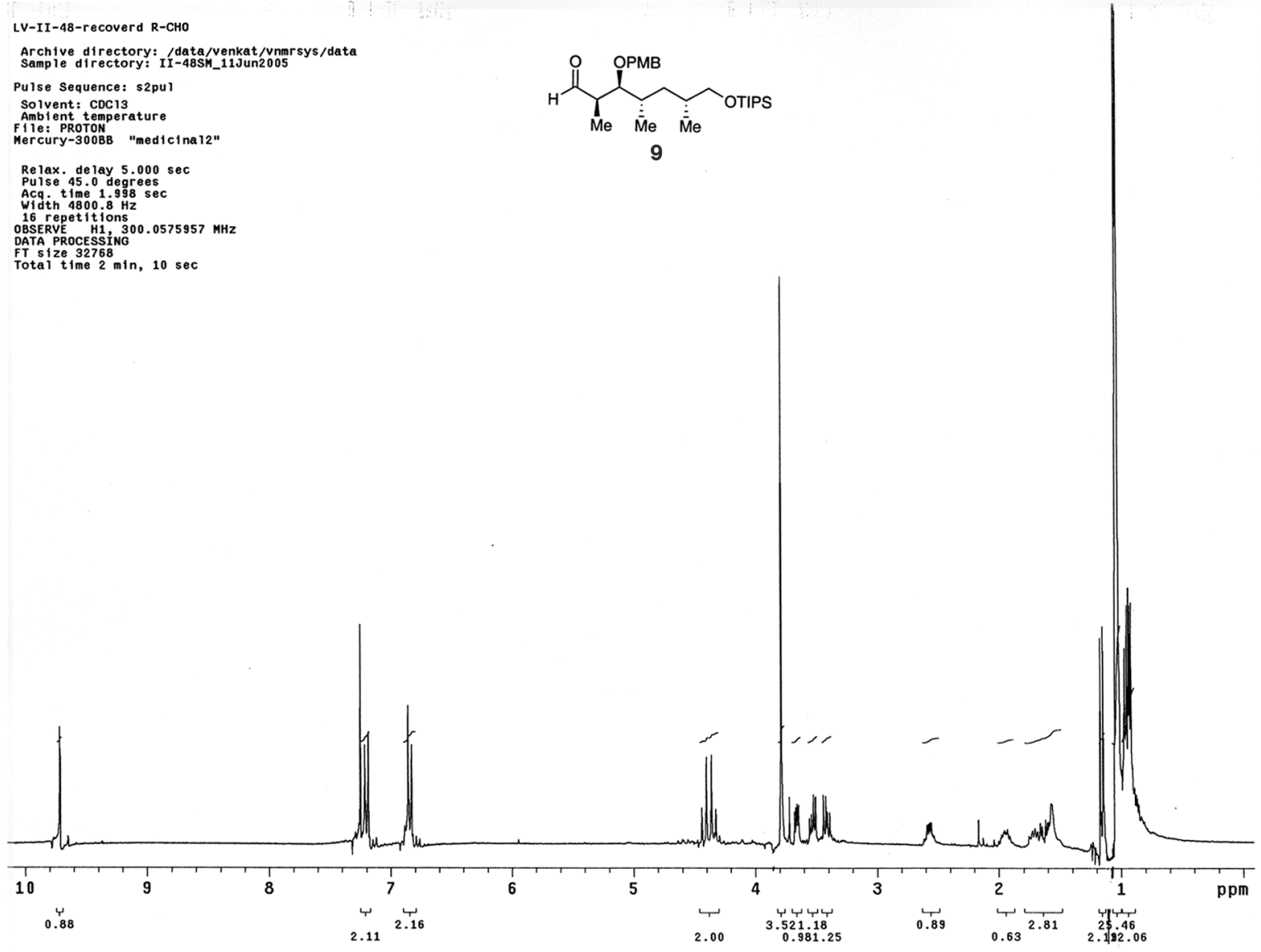

LV-II-48-recovered R-CHO

Archive directory: /data/venkat/unmrsys data
Sample directory: II-48SM_11Jun2005-10:51:54
File: CARBON

Pulse Sequence: s2pul

Solvent: ${ }^{\text {CDC13 }}$
Amb ient temperature
Mercury-300BB "medicinal1"

Relax. de lay $1.000 \mathrm{sec}$

Pulse 45.0 degrees

Acq time 1.815 soc

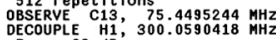

continuous $1 y$ on

DATA PROCESSING

FT size 131072

Total time $25 \mathrm{~min}, 5 \mathrm{sec}$
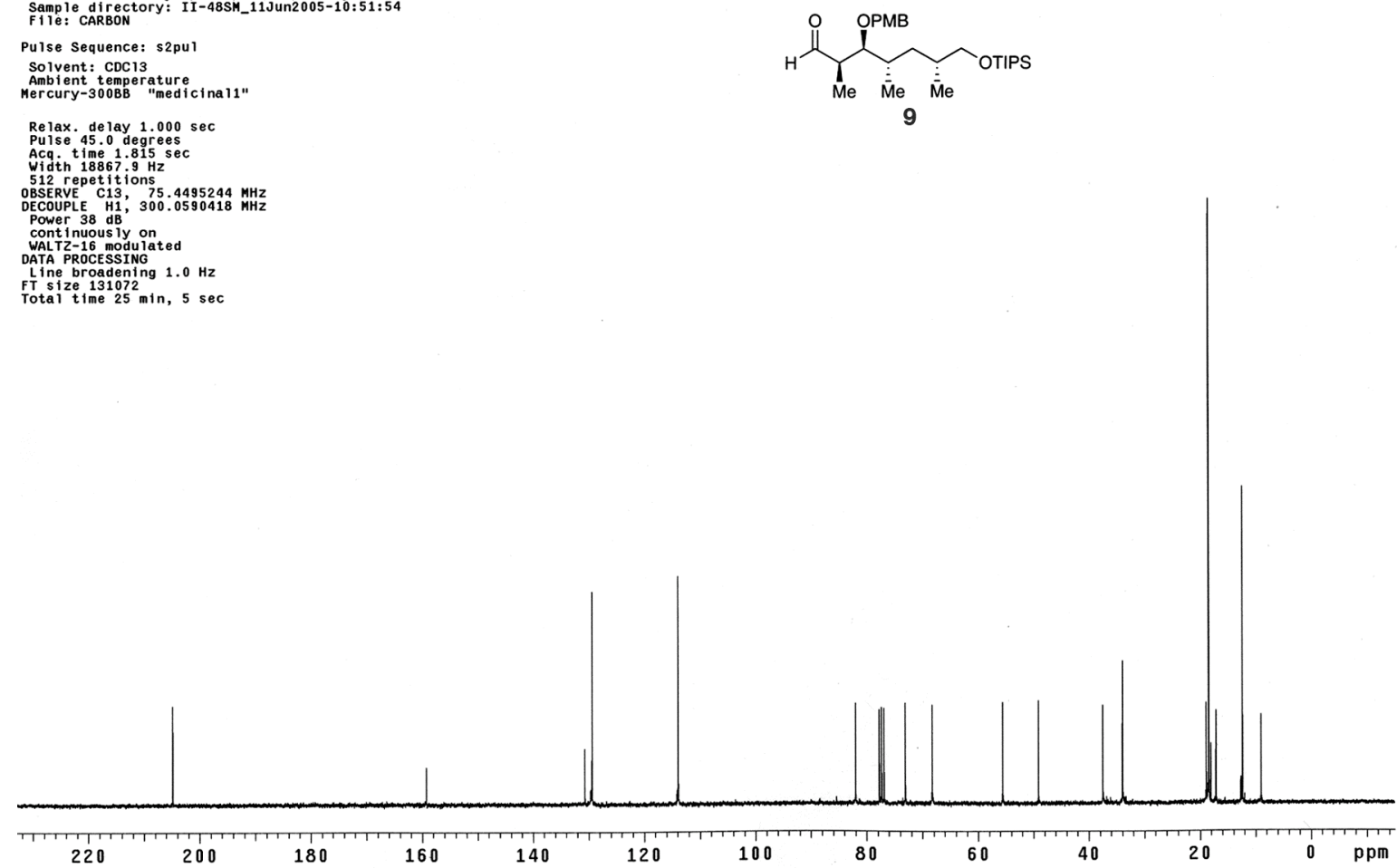
LV-II-48-a]dol

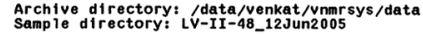

Pulse Sequence: s2pul

Solvent: CDC13

File: PROTON
Mercury-300BB "medicinal2"

Relax. de lay $5.000 \mathrm{sec}$

Acq. $t 1$ me 1.998 sec

Width 4800.8

OBSERVE H1, 300.0575957 MHz

DATA PROCESSING
FT S1Ze 32768
Total time
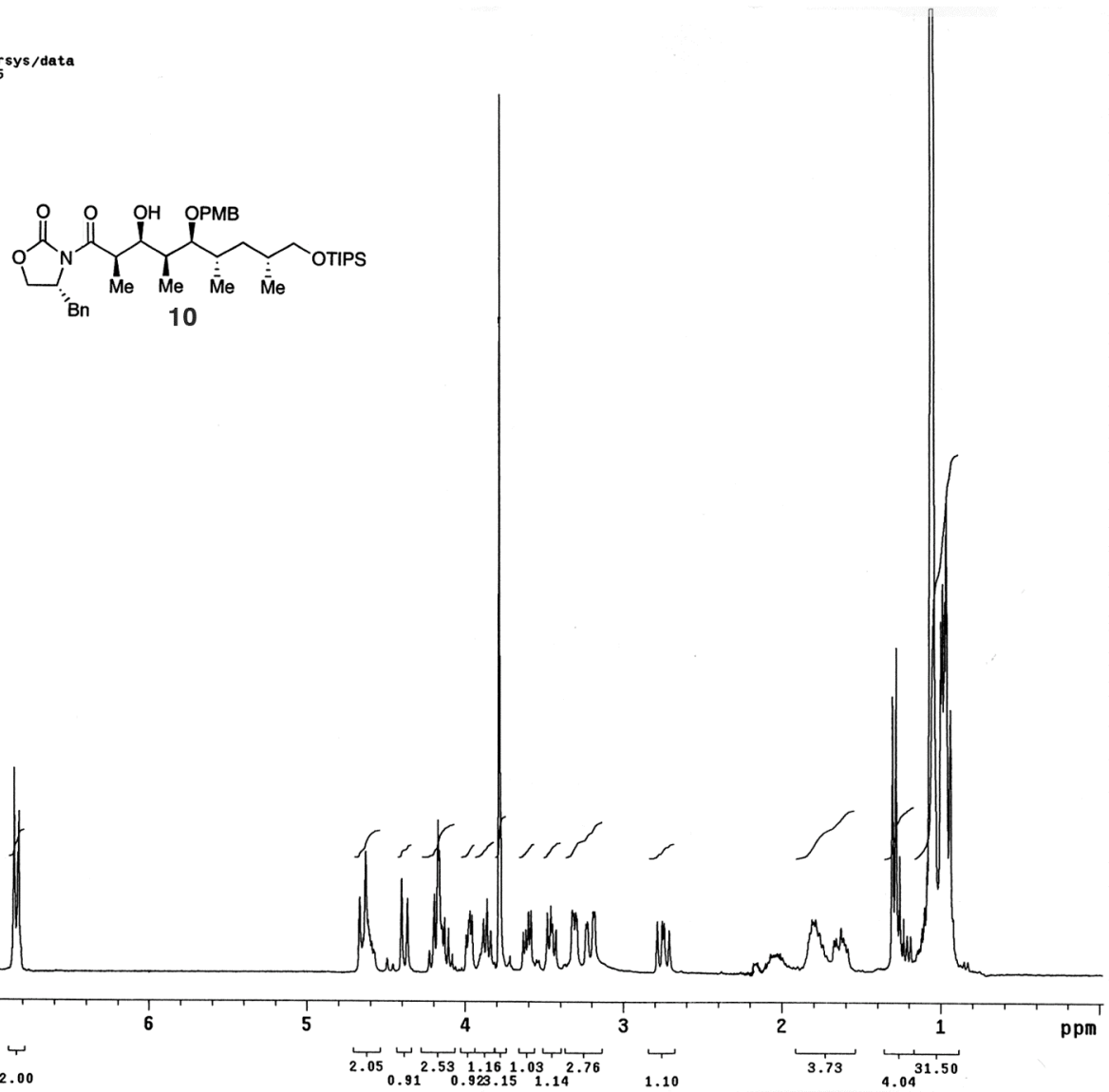

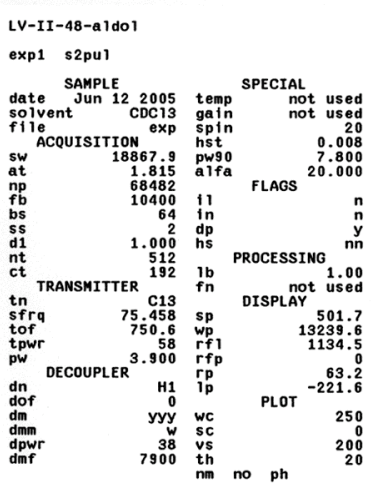
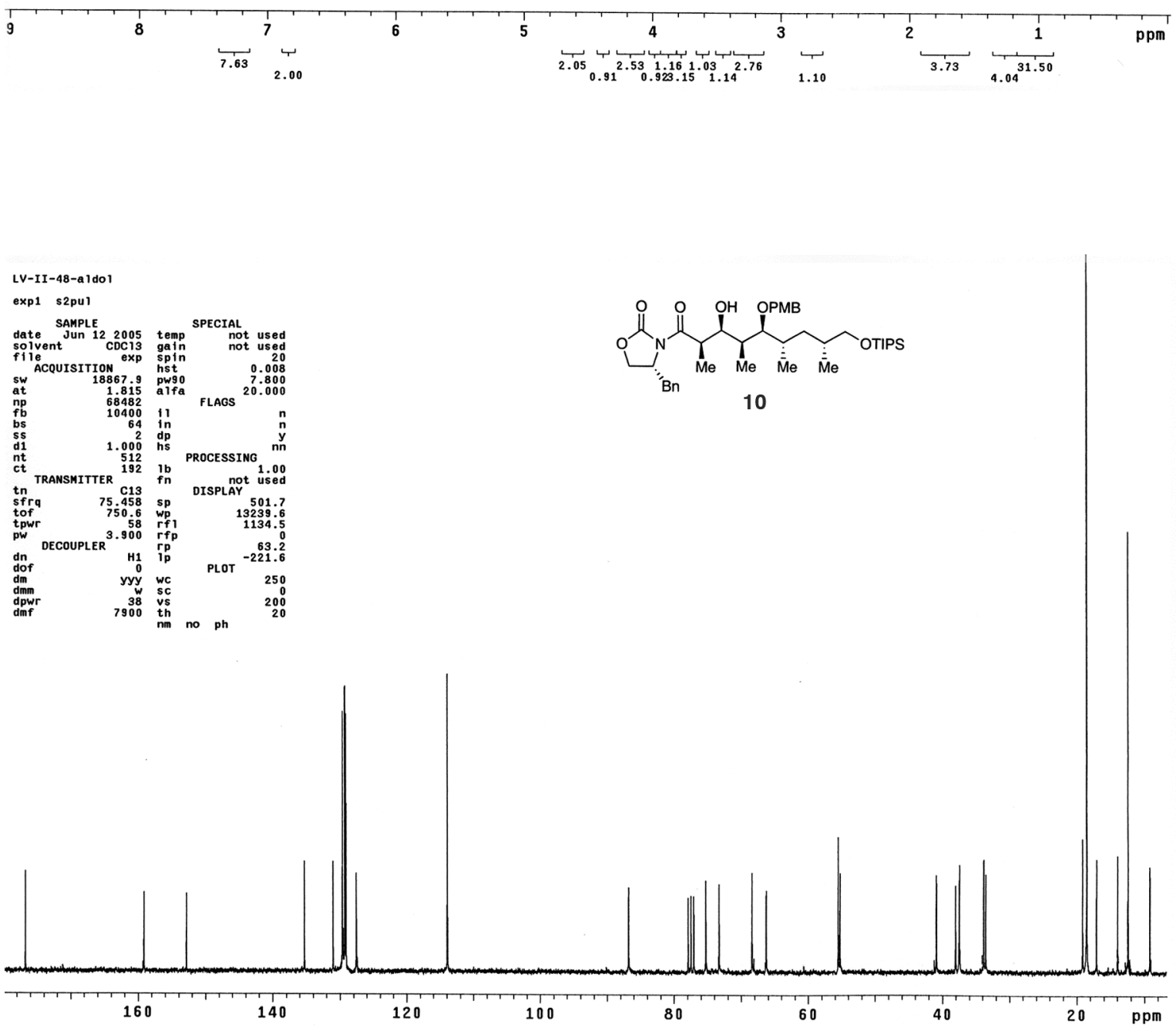

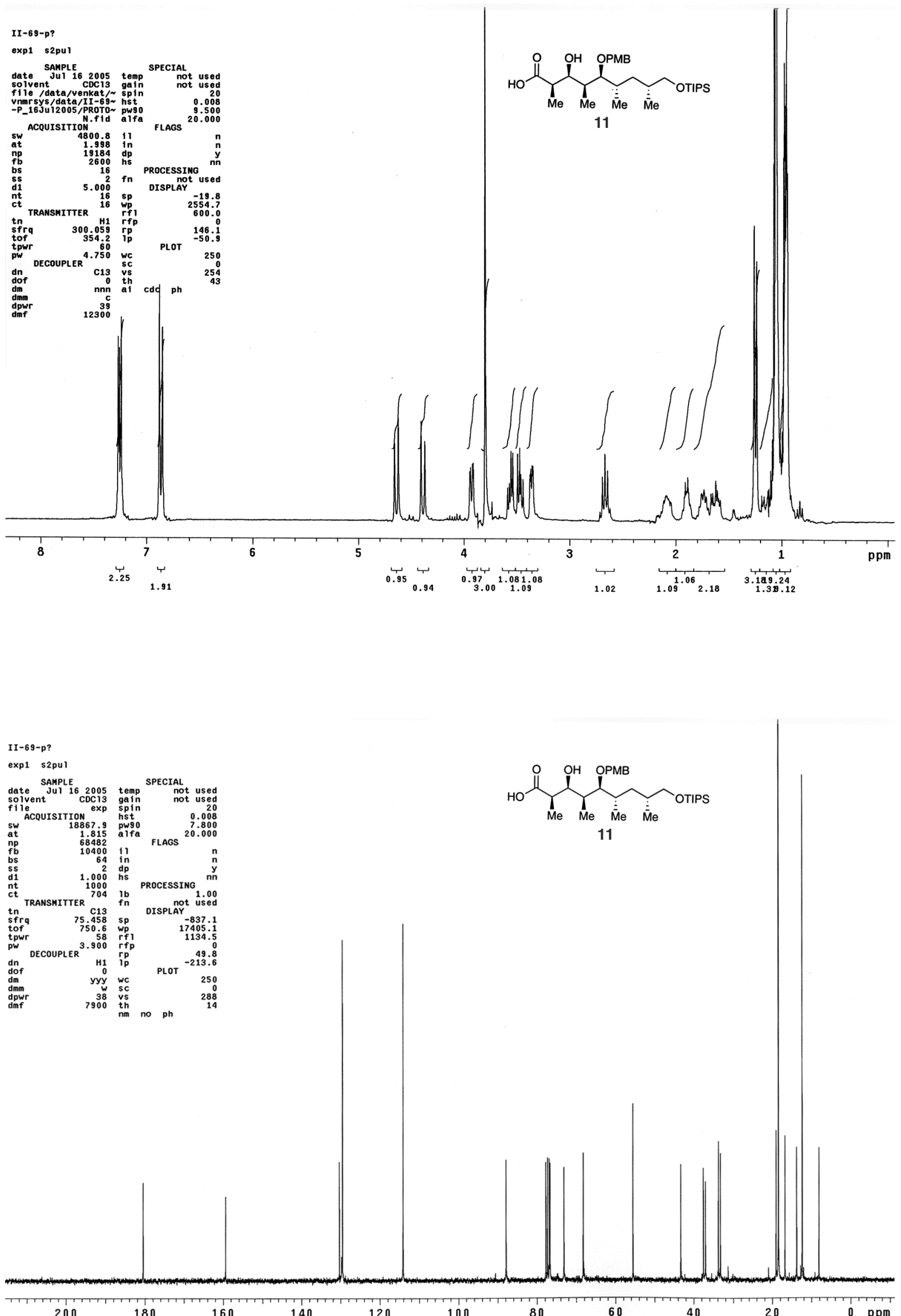
LV-II-52-F2

Archlve directory: /data/venkat/vnmisys/data
Sample directory: II $-52-\mathrm{F}_{2}$ 2_ $16 \mathrm{Jun} 2005$

Pulse Sequence: s2pur

Solvent: CDC13

Fercury-300BB "medicinal2"

Relax. de lay $5.000 \mathrm{sec}$
Pulse 45.0 degrees

Acg. $t$ time 1.998 sec

16 repetitions

OSERVE H1 300.0575957
OATA PROCESSINO

Total time $2 \mathrm{~min}, 10 \mathrm{sec}$

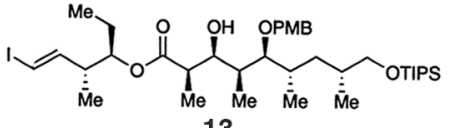

13

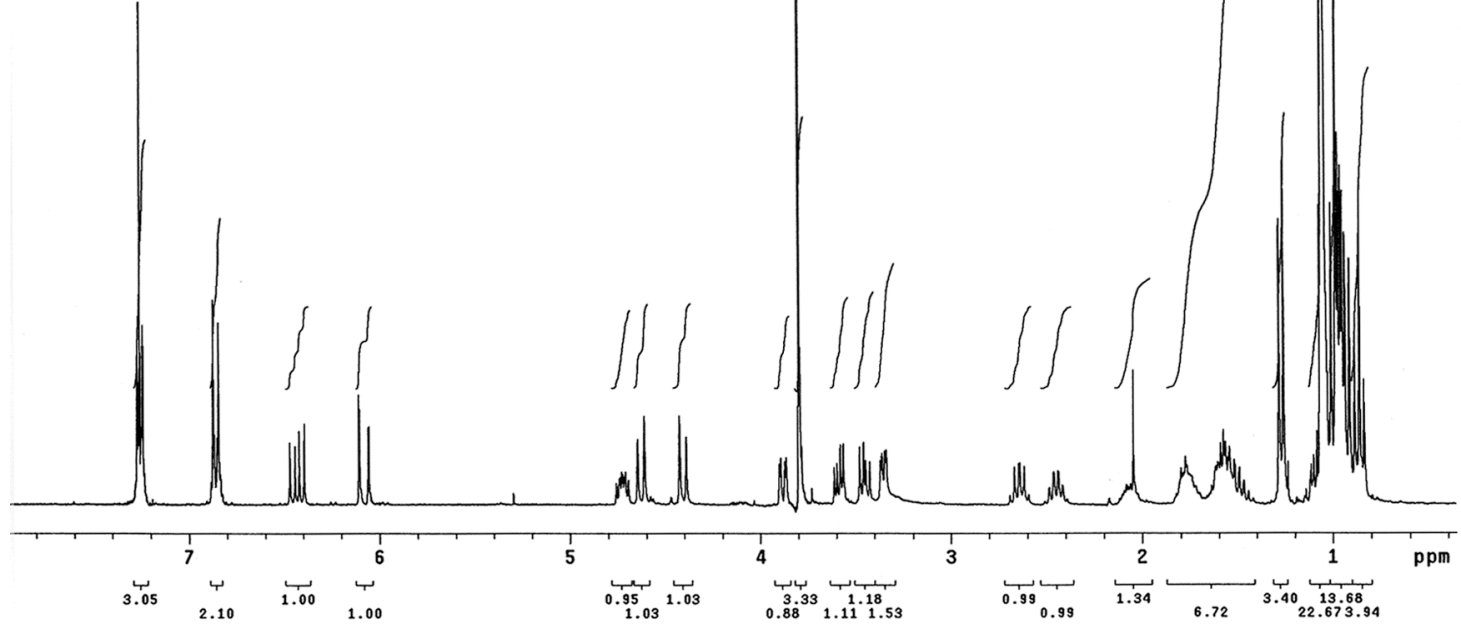

LV-II-52-F2

Archive directory: /data/venkat/vnmrsys/data
Sample directory: II-52-F2_18Junz005

Pulse Sequence: s2pul

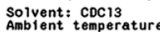

File: CARBON
Mercury-300B "medicinal2"

Relax. delay $1.000 \mathrm{sec}$

Pcse 4 ime 1.815 se

OBSERVE C13, 75.4495244 MHZ
OECOUPLE H1 300.0590418 HHZ

Power 38 dB
Cont nuous on
walt 7 - 16 modulated

Line broadening $1.0 \mathrm{~Hz}$

FT s size $13107221.0 \mathrm{~Hz}$
Total time $25 \mathrm{~m} / \mathrm{n}, 5 \mathrm{sec}$
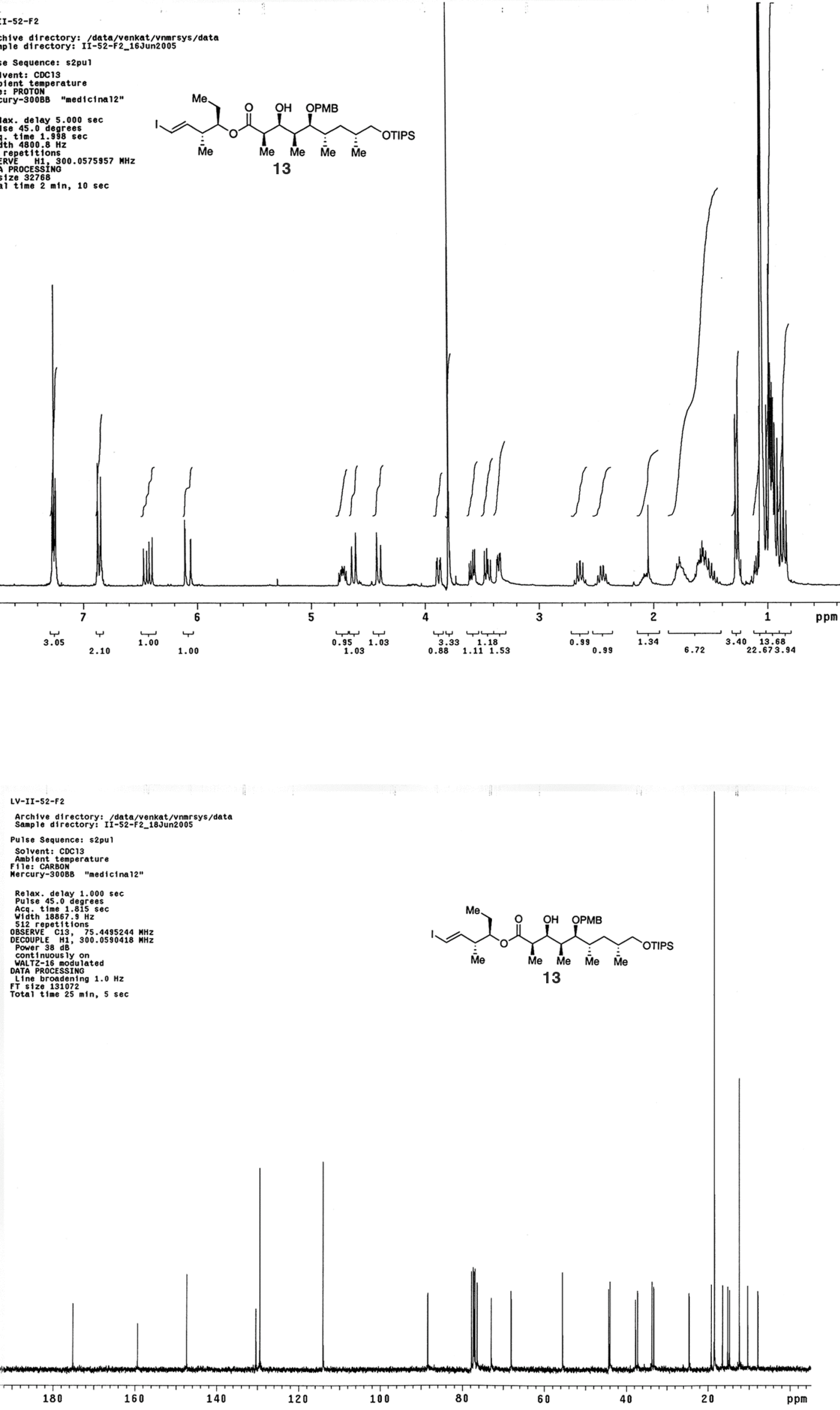


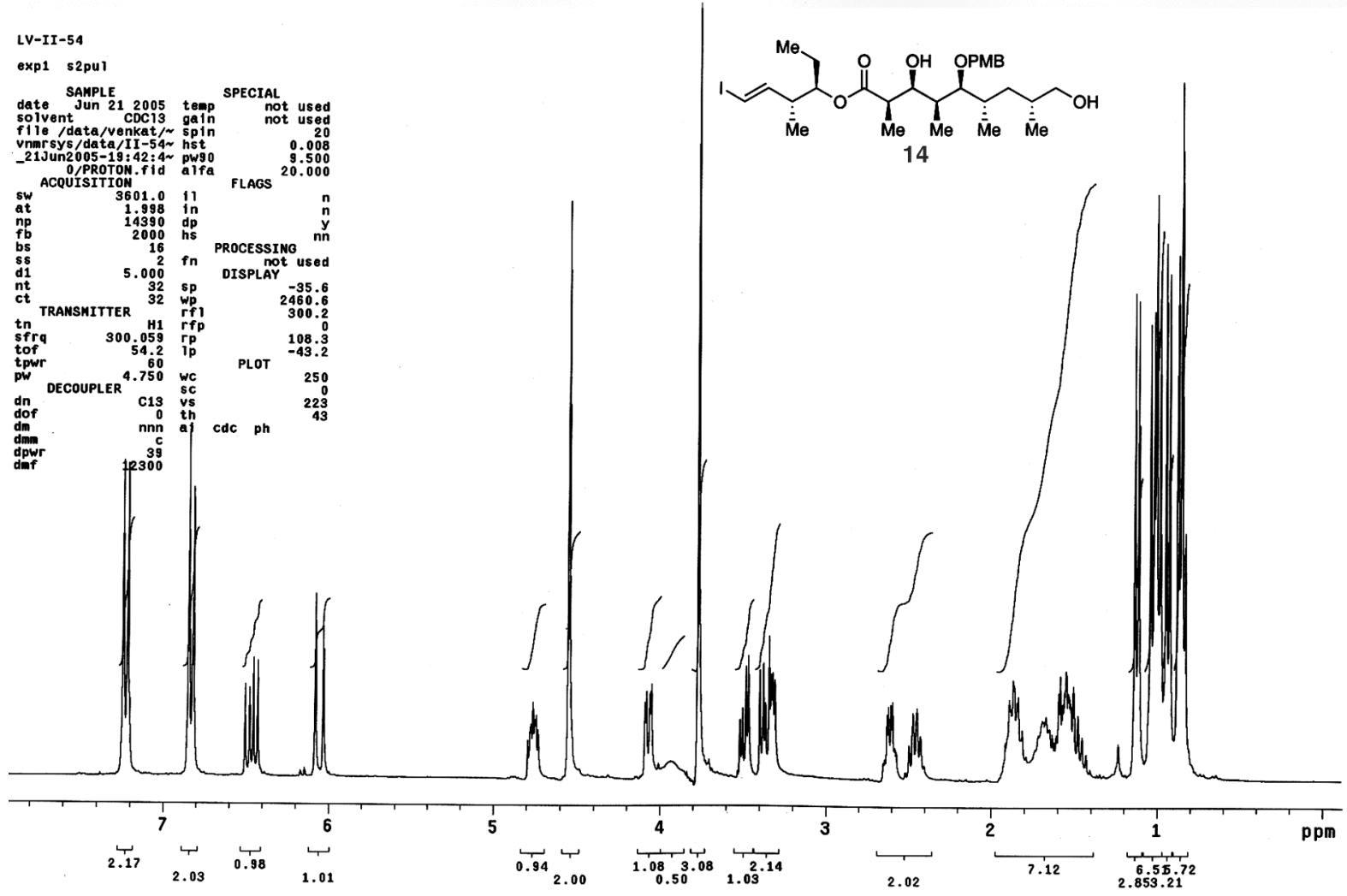

LV-II-54

Archive directory: /data/venkat/unmrsys/data

Archile directory: II-54_21Jun2005
File: CARBON

Pulse Sequence: s2pu1

Solvent: $\operatorname{CDC13}$

Ambient temperature
Mercury-300BB "medicinal1"

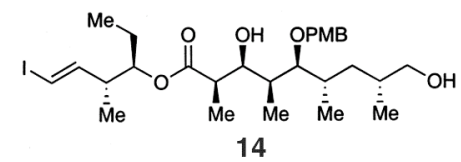

Relax. delay $1.000 \mathrm{sec}$
Pulse 45.0 degrees

Acq. $t$ ime 1.815 sec

OBSERVE C13, 75.4495244 MHZ

OECOUPLE H1, 300.0590418 MHZ

Power $38 \mathrm{~dB}$

WALTZ-16 modu 1 ated
DATA PROCESSING

Line broadening $1.0 \mathrm{~Hz}$

Total time $48 \mathrm{~min}, 47 \mathrm{sec}$

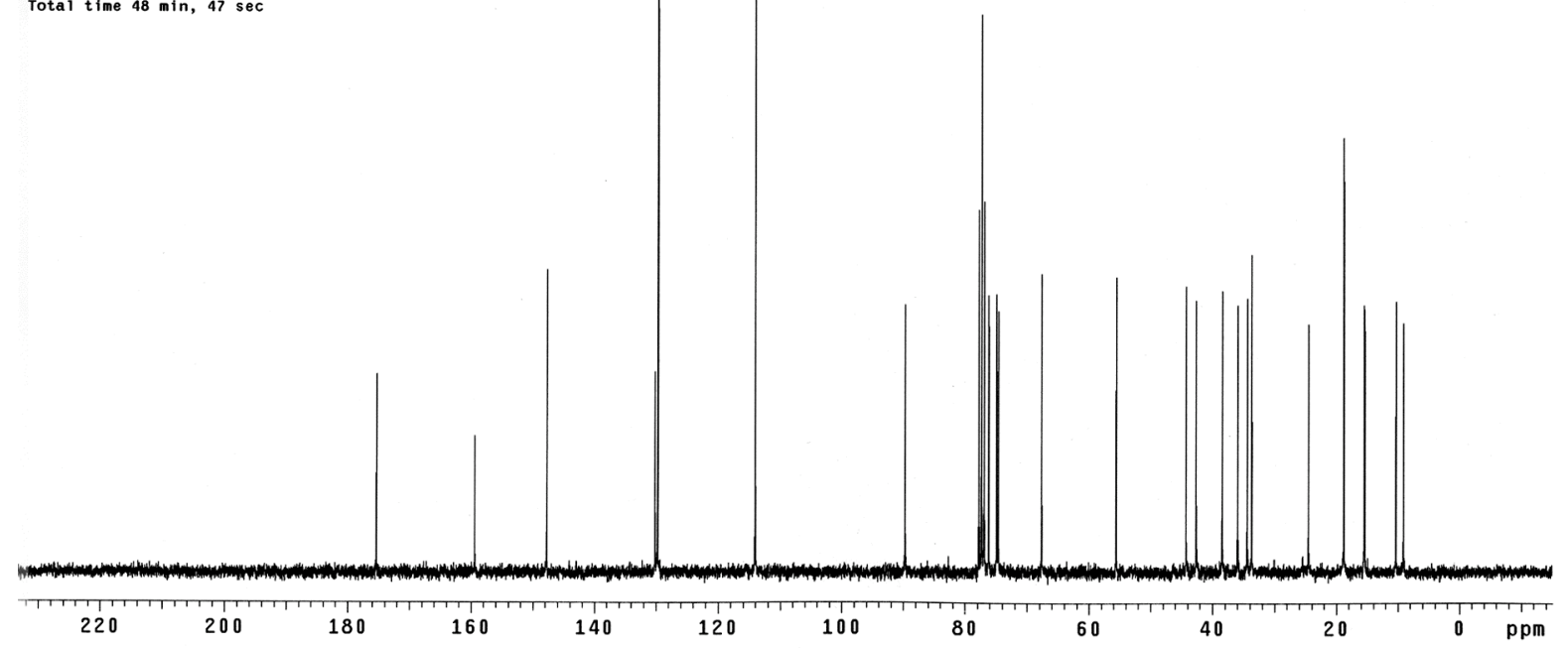


iv-II-76 (aldehyde)

Archive directory: /data/venkat/vnmmsys/data
Sample directory: II-76_26Ju12005

Pulse Sequence: s2pul

Solvent: ${ }_{\text {CDCl3 }} 3$
Ambient temperature

File: PROTON
Mercury-300B "medicinal2"

Re lax. de lay $5.000 \mathrm{sec}$

Pulse 45.0 degrees
Acq. time 1.998 sec

Width $4800.8 \mathrm{~Hz}$

16 repet itions
OBSERVE H1, 300.0575957 MHZ

DATA PROCESSING

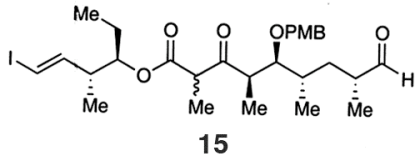

15

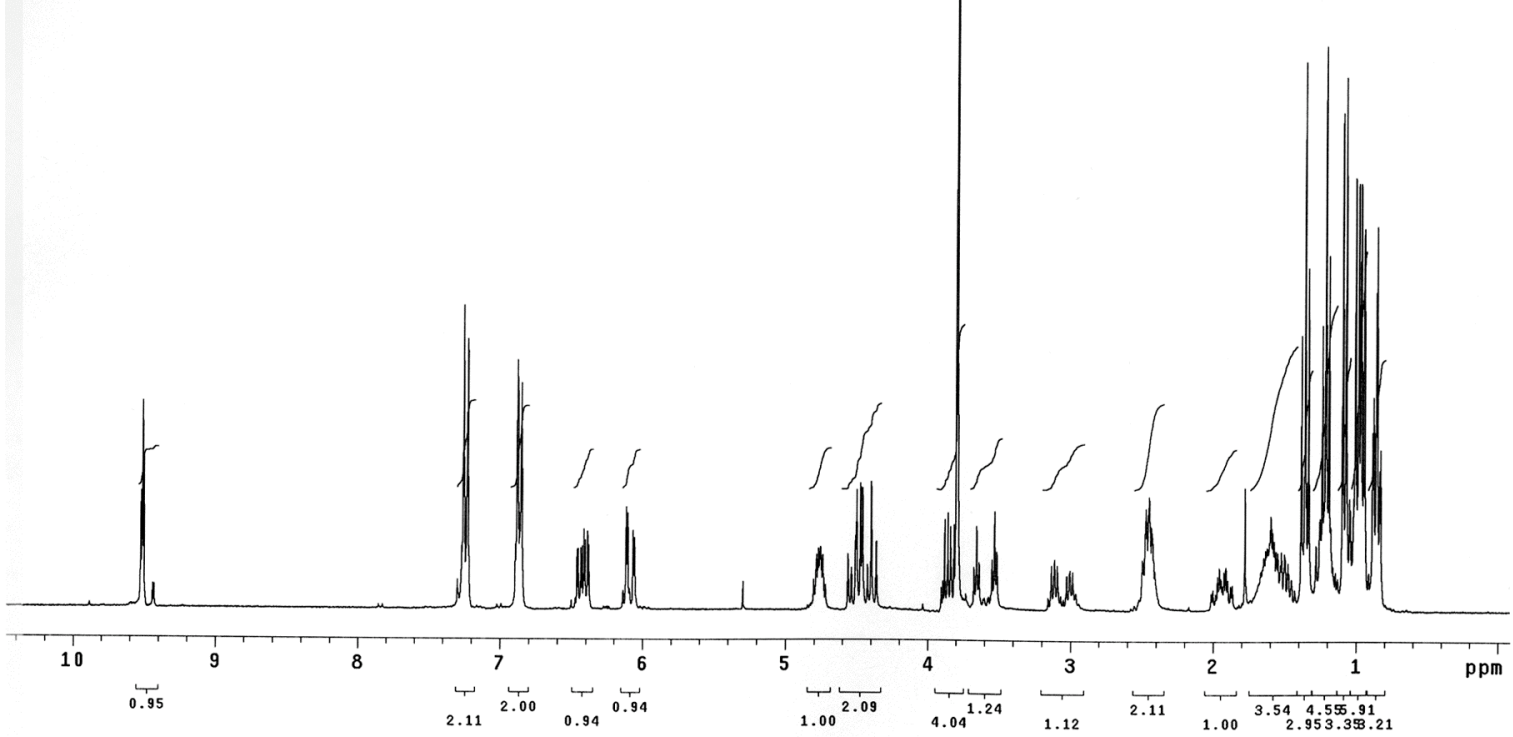

LV-II-58 (a)dehyde)

Archive directory: /data/venkat/unmrsys/data
Sample directory: II-58_26Jun2005
File: CARBON

Pulse Sequence: s2pur

Solvent: $\operatorname{COC}_{13}$

Amblent temperature
Mercury-300BB "medicina11"

Relax. de lay $1.000 \mathrm{sec}$

Acq. time $1.815 \mathrm{sec}$

OBSERVE C13, 75.4495244 MH

Power $38 \mathrm{~dB}$,
cont inuous ly on

cont inuous ly on
WAL $2-16$ modulated

Line broadening $1.0 \mathrm{~Hz}$

FT size 131072 in, $47 \mathrm{sec}$
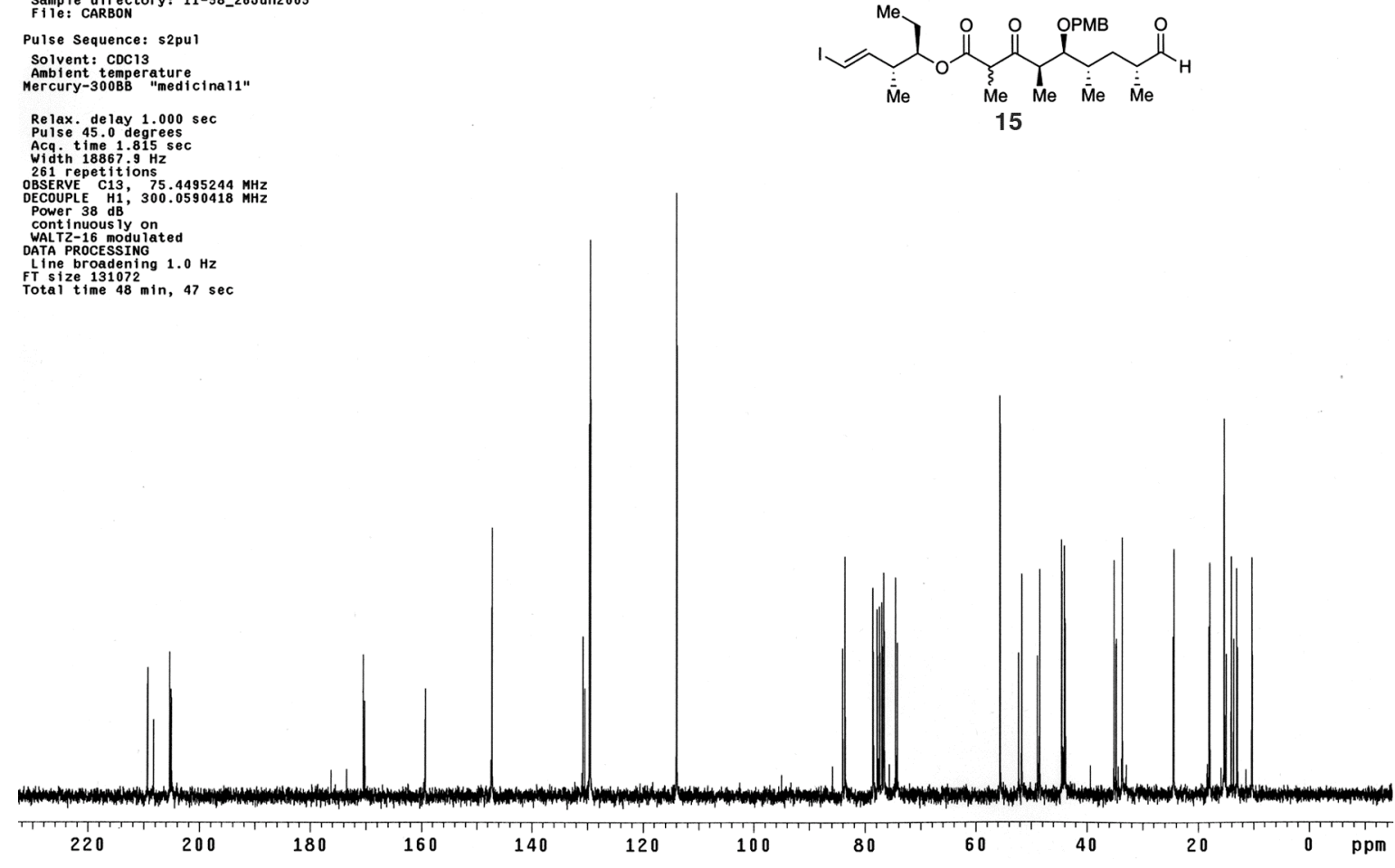


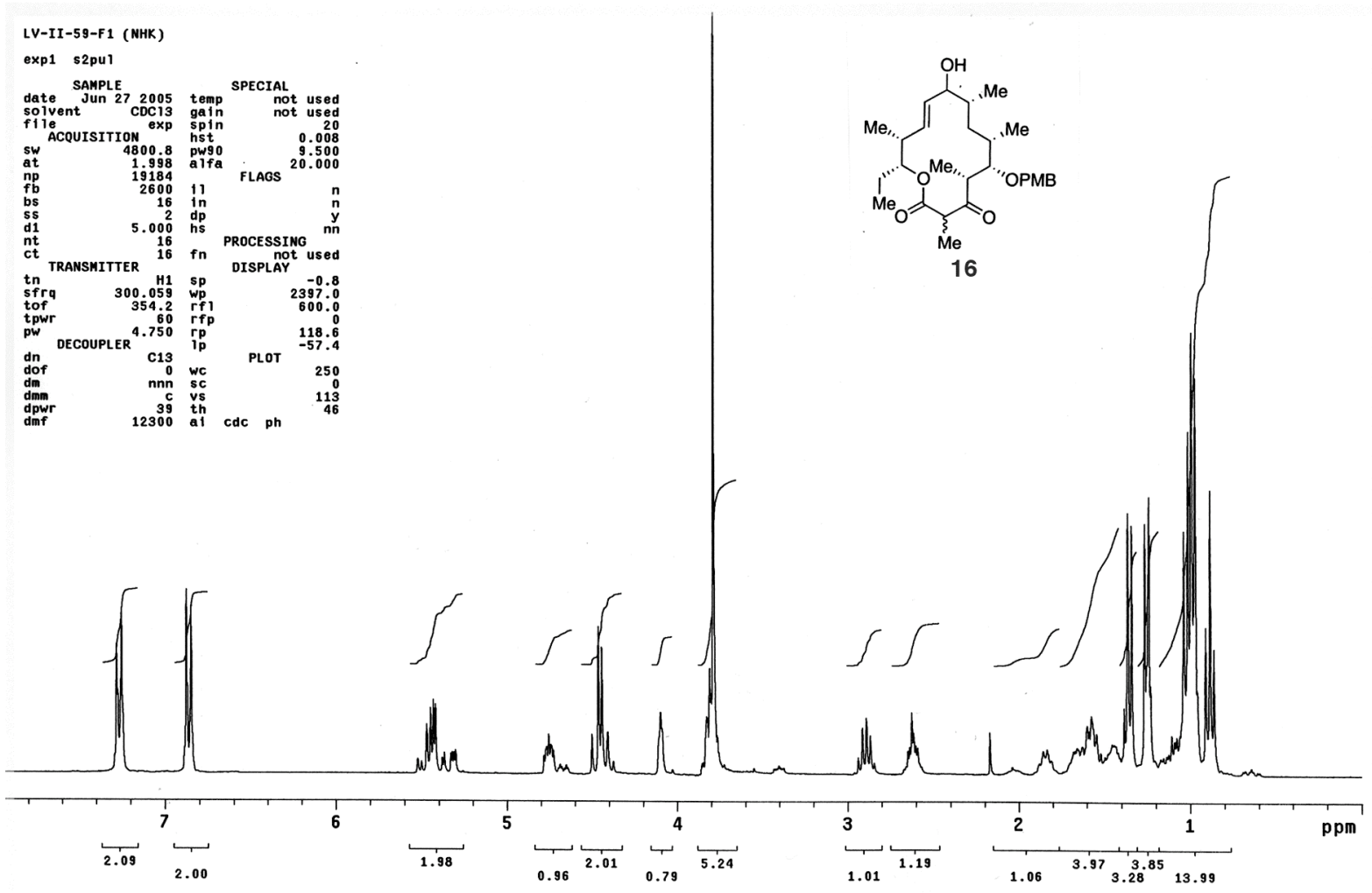

LV-II-59-F 1 (NHK)

Archive directory: /data/venkat/vnmrsys/data

Pulse Sequence: s2pul

Solvent: $\mathrm{CDC}_{13}$

Ambient temperature

Relax. delay $1.000 \mathrm{sec}$
Pulse 45.0 degrees

Pulse 45.0 degrees
Acq. time 1.815
sec

Acq. time 1.815 s

OBSERVE C13, $75.4495244 \mathrm{MHZ}$

DECOUPLE H1, 300.0590418 MHZ

Power 38 dB
continuous ly on
walT

DATA PROCESSING

TT size $1310721.0 \mathrm{~Hz}$
Total time $1 \mathrm{hr}, 37 \mathrm{~min}, 21 \mathrm{sec}$
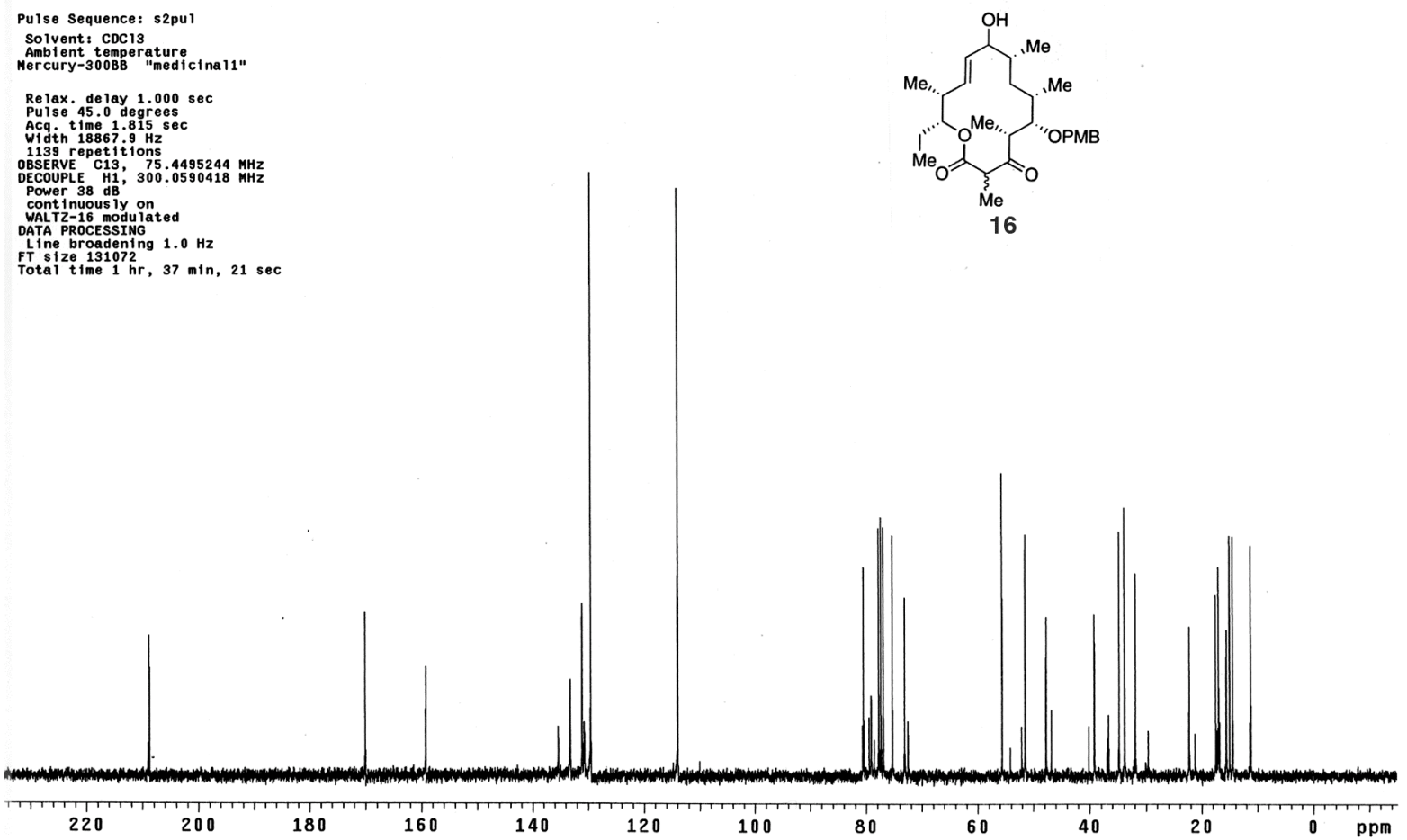
LV-II-64 (Dess-Martin)

Archive directory: /data/venkat/vnmrsys/dat

Pulse Sequence: s2pul

Solvent: CDC13
Ambient temperature

File: PROTON
Mercury-300B "medicinal2"

Relax. delay $5.000 \mathrm{sec}$

Acq. time 1.998 sec

Width 4800.8

OBSERVE H1, 300.0575957 MHZ

FT size 32768
Total time $2 \mathrm{~min}, 10 \mathrm{sec}$

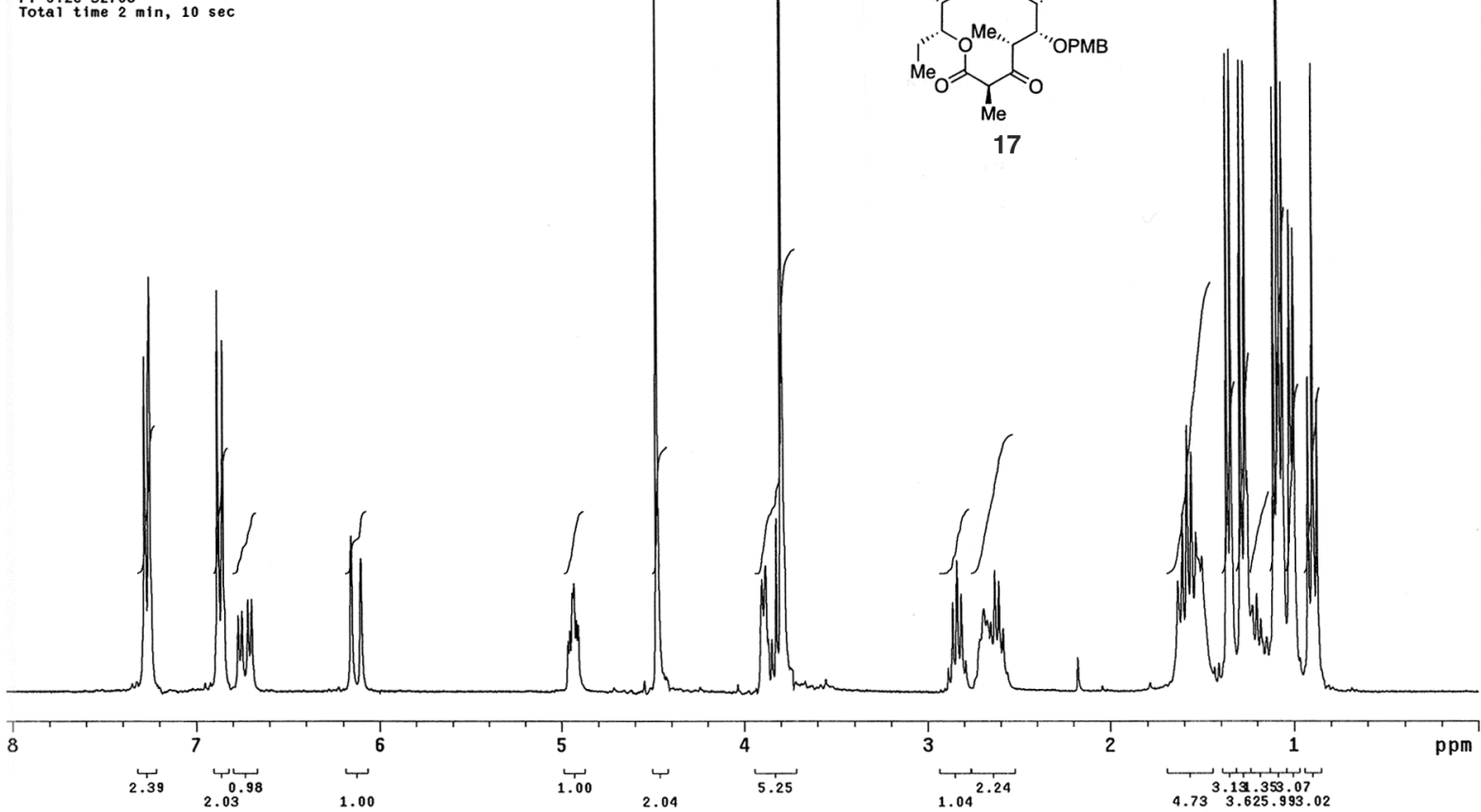

LV-II-64

Archive directory: /data/venkat/ynmrsys/data
Sample directory: II-64_01Ju12005-20:26:04

Pulse Sequence: s2pul

Solvent: $\mathrm{CDCl}_{3}$

File: CARBON "medicinalo

Relax. de lay $1.000 \mathrm{sec}$

Pulse 45.0 degrees

Width $18867.9 \mathrm{~Hz}$

200 VE C13, $75.4495244 \mathrm{MHz}$
DECOUPLE H1, $300.0590418 \mathrm{MHz}$

Power $38 \mathrm{~dB}$

continuous iy on

DATA PROCESSING $1.0 \mathrm{H}$

Line broadening $1.0 \mathrm{~Hz}$
FT size 131072
Total time $1 \mathrm{hr}, 37 \mathrm{~m} 1 \mathrm{n}, 21 \mathrm{sec}$ 

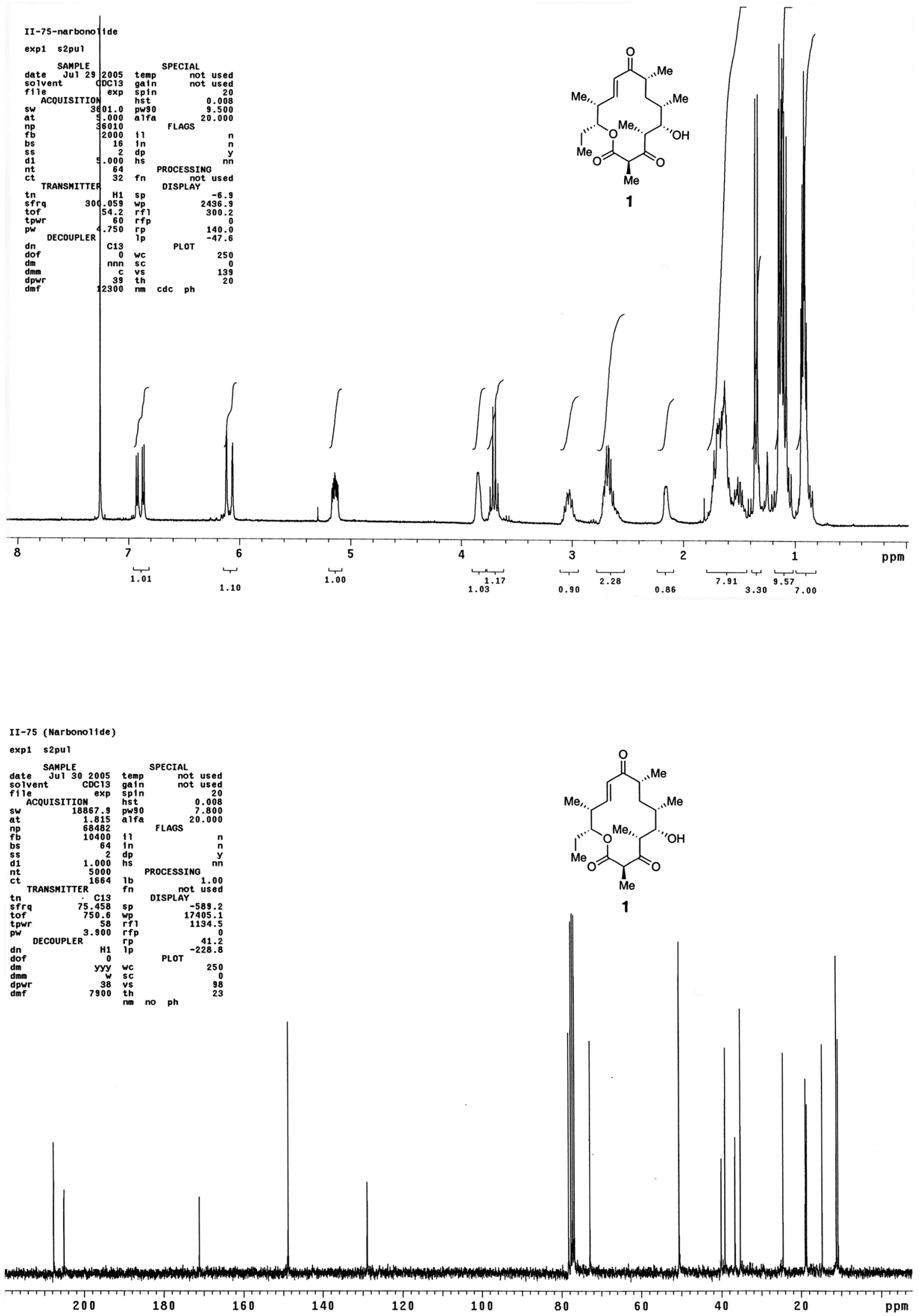

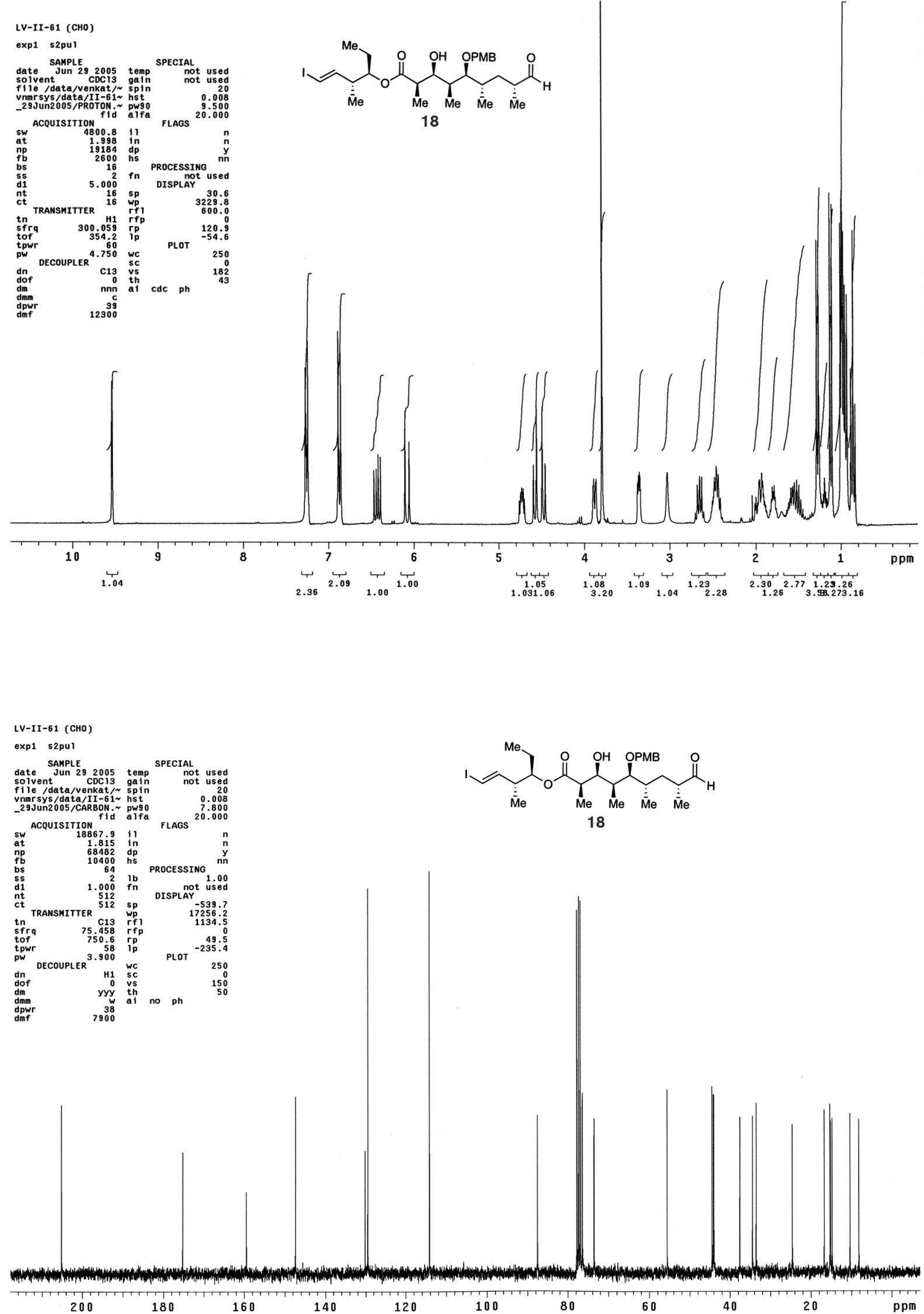
LV-II-62-F 1p

Archive directory: /data/venkat/vmm sys/data

Pu1se Sequence: s2pur

Solvent: $\mathbf{C D C 1 3}$

Amb ent temp
File: PROTON

"medicina12"

Relax. delay $5.000 \mathrm{sec}$

Acq. $t$ time 1.998 sec

16 repetitions

OBSERVE H1 300.0575957 MHZ

FT s $12 e 32768$
Total time $2 \mathrm{~min}, 10 \mathrm{sec}$

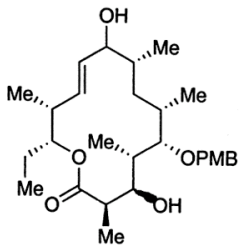

19

(major epimer)

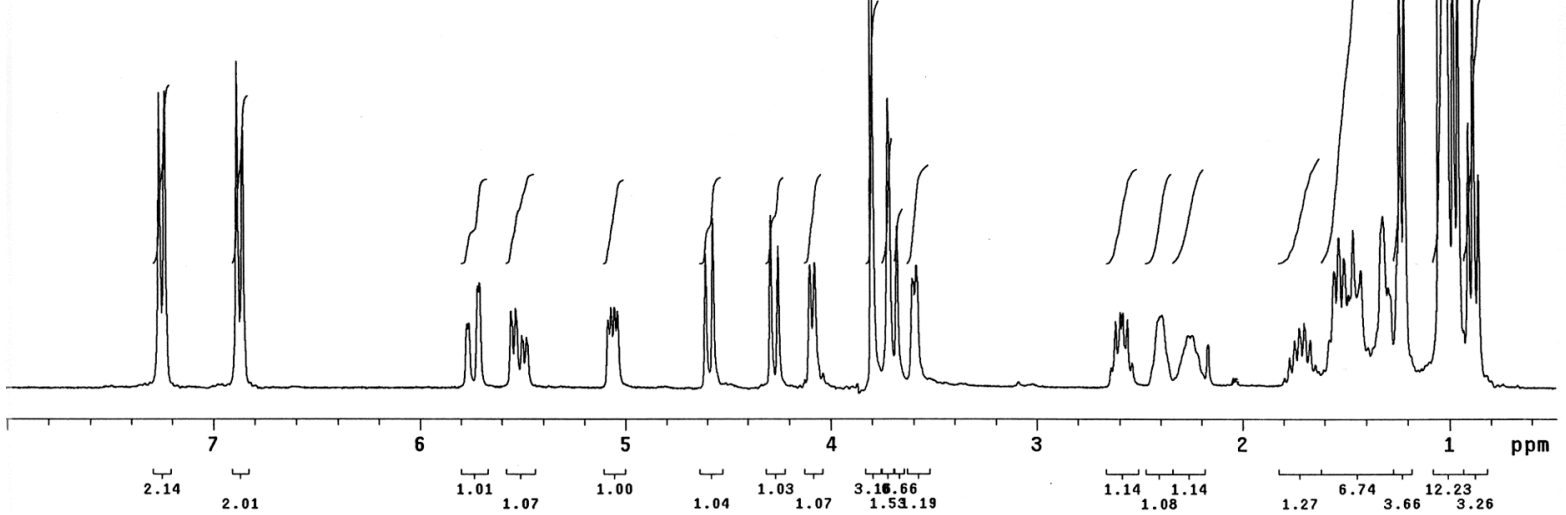

LV-II-62-F 1p

Archive directory: /data/venkat/unmrsys/data
Sample directory: II $-62-\mathrm{F}_{1}{ }_{1} 30 \mathrm{Jun} 2005$

Pulse Sequence: s2pul

Solvent: $\operatorname{CDC}_{13}$

Ambi ent temperature
Mercury-300BB "medicinal1

Re lax. de lay $1.000 \mathrm{sec}$

Acq. time $1.815 \mathrm{se}$

Widin $18867.9 \mathrm{~Hz}$

OBSERVE C13, $75.4495244 \mathrm{MHZ}$

DECOUPLE H1, $300.0590418 \mathrm{MHZ}$

Power 38 dB

WAL TZ-16 modu 1 ate

DATA PROCESSING $1.0 \mathrm{~Hz}$

FT size 131072 . $37 \mathrm{~min}, 21 \mathrm{sec}$ 

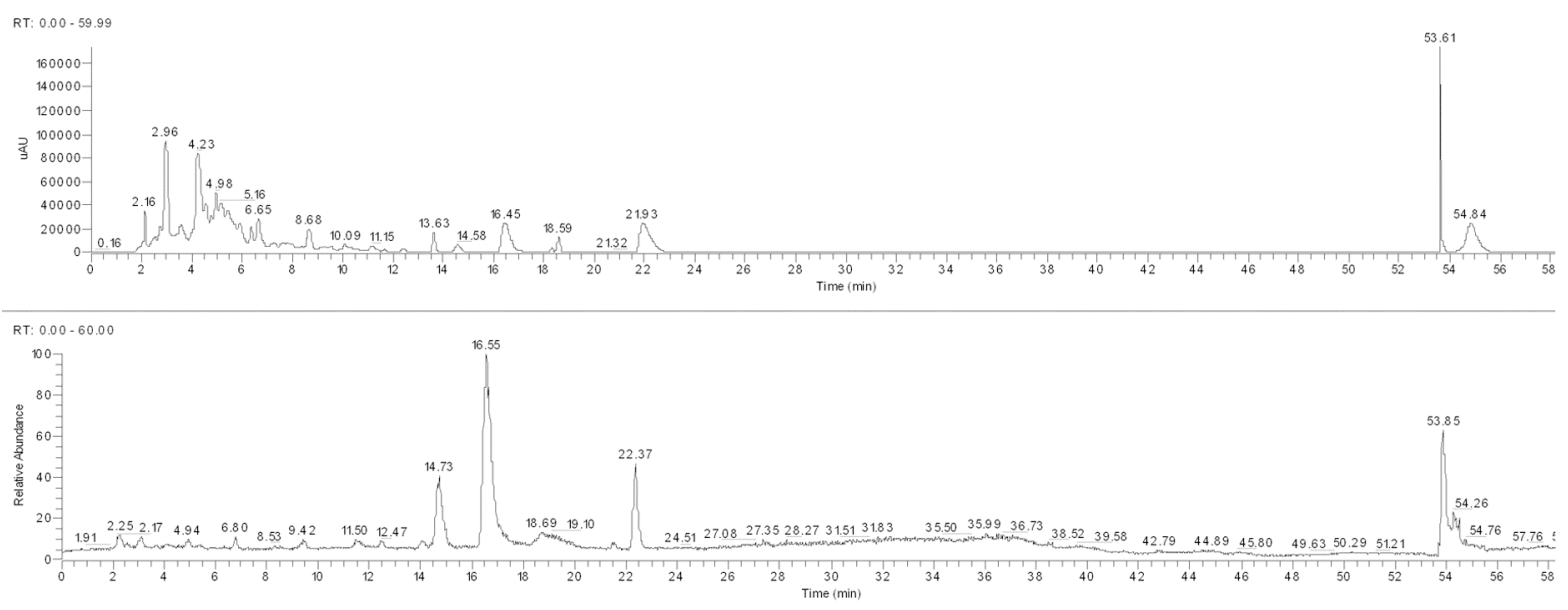

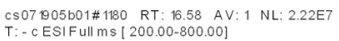

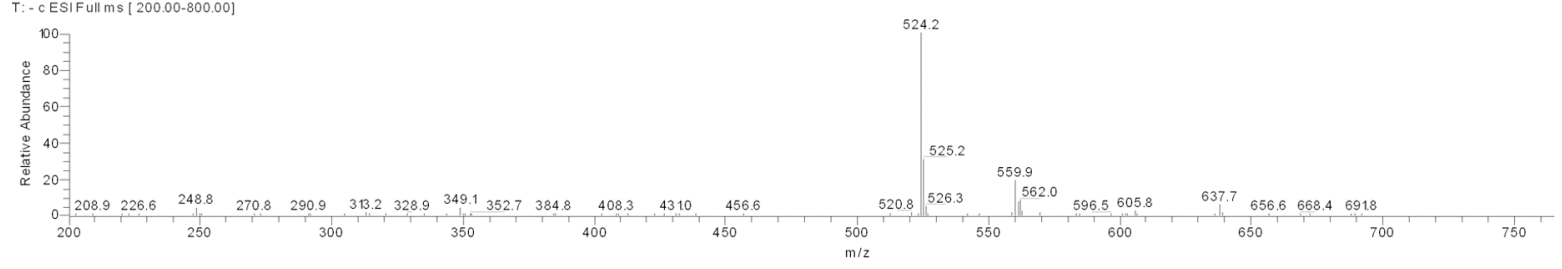

LC-MS trace of EtOAc extract from the crude fermentation broth after incubation of narbonolide with $S$. venezuelae pikAI deletion mutant BB138. LC peak at 16.45 min (top frame) corresponds to MS (ESI +) chromatogram peak at 16.55 (middle frame), with an extracted ion mass of $524\left(\mathrm{M}-\mathrm{H}^{+}\right)$(bottom frame).

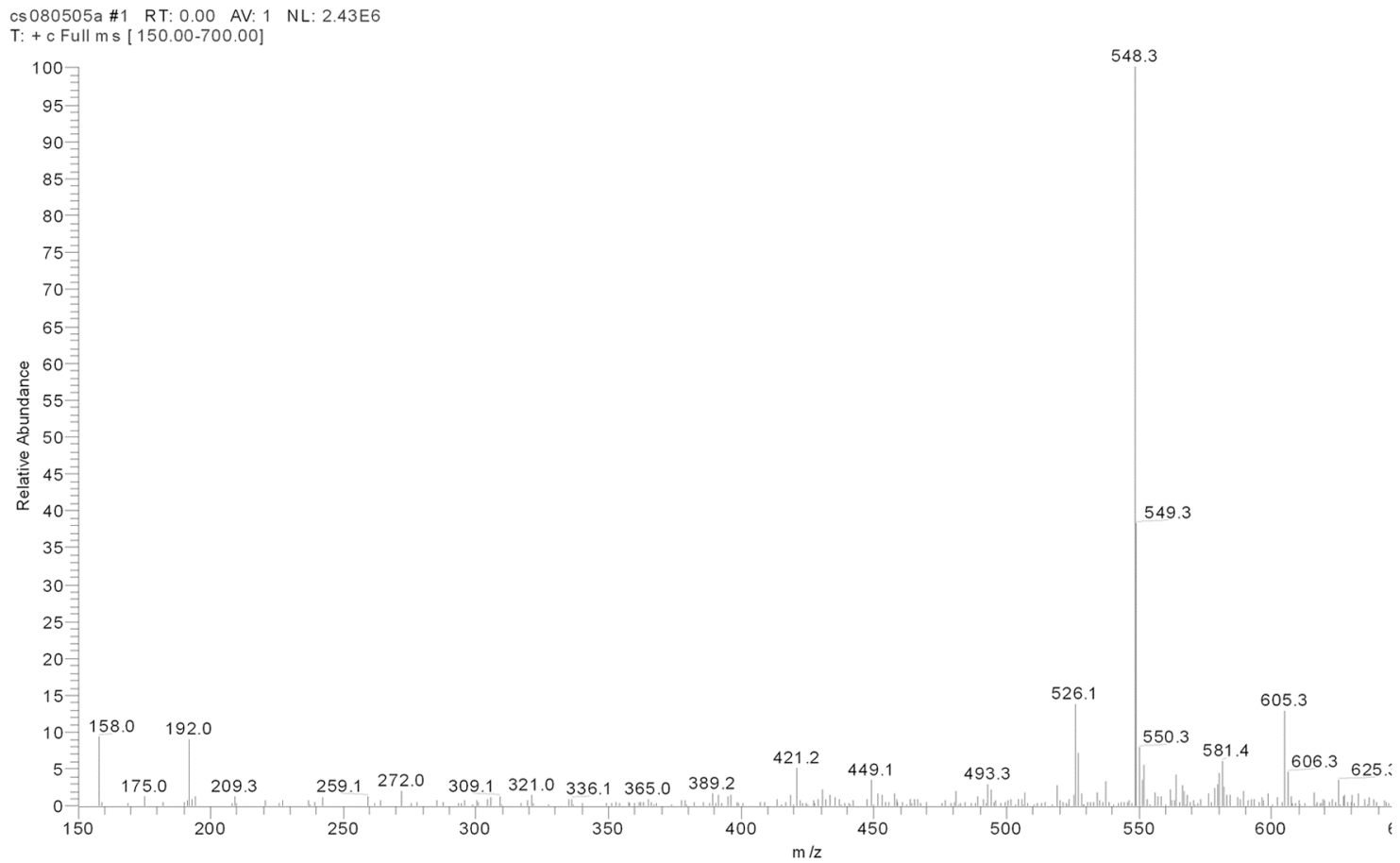

MS (ESI -) of pikromycin peak (10.4 min) collected from HPLC purification of narbonolide biotransformation: $548\left(\mathrm{M}^{+}+\mathrm{Na}\right), 526\left(\mathrm{M}^{+}+\mathrm{H}^{+}\right)$. 


\section{References}

(1) Dess, D. B.; Martin, J. C. J. Am. Chem. Soc. 1991, 113, 7277-7287.

(2) Ireland, R. E.; Liu, L. J. Org. Chem. 1993, 58, 2899.

(3) Akey, D. L.; Kittendorf, J. D.; Giraldes, J. W.; Fecik, R. A.; Sherman, D. H.; Smith, J. L. Nat. Chem. Biol. 2006, 2, 537-542.

(4) Pilli, R. A.; de Andrade, C. K. Z.; Souto, C. R. O.; de Meijere, A. J. Org. Chem. 1998, $63,7811-7819$. 